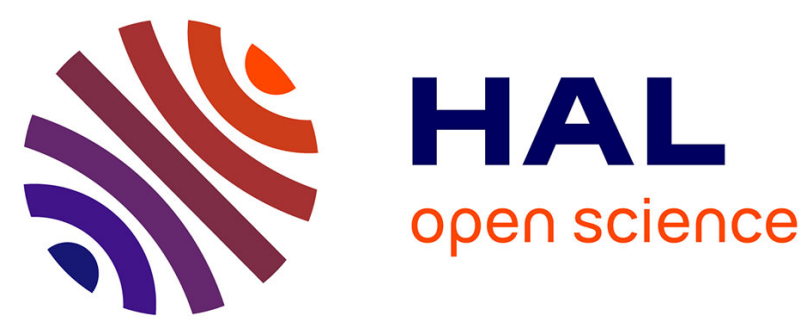

\title{
Closed-form solutions for predicting the thick elastic plate behavior of CLT and timber panels with gaps
}

Arthur Lebée, Gilles Forêt, Florent Lyon, Lorenzo Franzoni

\section{To cite this version:}

Arthur Lebée, Gilles Forêt, Florent Lyon, Lorenzo Franzoni. Closed-form solutions for predicting the thick elastic plate behavior of CLT and timber panels with gaps. Engineering Structures, 2018, 164, pp.290-304. 10.1016/j.engstruct.2018.02.073 . hal-01978742

\section{HAL Id: hal-01978742 \\ https://hal-enpc.archives-ouvertes.fr/hal-01978742}

Submitted on 11 Jan 2019

HAL is a multi-disciplinary open access archive for the deposit and dissemination of scientific research documents, whether they are published or not. The documents may come from teaching and research institutions in France or abroad, or from public or private research centers.
L'archive ouverte pluridisciplinaire HAL, est destinée au dépôt et à la diffusion de documents scientifiques de niveau recherche, publiés ou non, émanant des établissements d'enseignement et de recherche français ou étrangers, des laboratoires publics ou privés. 


\title{
Closed-form solutions for predicting the thick elastic plate behavior of CLT and timber panels with gaps
}

\author{
L. Franzoni ${ }^{1}$, A. Lebée ${ }^{1 *}$, F. Lyon ${ }^{2}$, G. Forêt ${ }^{1}$ \\ ${ }^{1}$ Laboratoire Navier, UMR 8205, IFSTTAR, CNRS, UPE \\ École des Ponts ParisTech, 6 et 8 avenue Blaise Pascal \\ 77455 Marne-la-Vallée cedex-2, France \\ ${ }^{2}$ Centre Scientifique et Technique du Batiment (CSTB) \\ 84 Avenue Jean Jaurès, 77447 Marne-la-Vallée cedex-2, France \\ *e-mail: arthur.lebee@enpc.fr
}

\begin{abstract}
In a former paper by the authors [1], the elastic behavior of Cross Laminated Timber (CLT) and timber panels having periodic gaps between lateral lamellae has been analyzed. A thick plate homogenization scheme based on Finite Elements computations has been applied. The predicted behavior was in agreement with experimental results. In this paper, simplified closed-form solutions are derived in order to avoid FE modeling. Both cases of narrow gaps of CLT panels and wide gaps of innovative lightweight panels are investigated. CLT and timber panels with gaps are modeled as a space frame of beams connected with wooden blocks. The contribution of both beams and blocks to the panel's mechanical response is taken into account, leading to closed-form expressions for predicting the panel's stiffnesses and maximum longitudinal and rolling shear stresses. The derived closed-form solutions are in agreement with the reference FE results and they can be used for practical design purposes.
\end{abstract}

Keywords: Cross Laminated Timber, Timber panels with gaps, Thick plate theory, Homogenization, Engineered timber products

\section{Introduction}

Cross Laminated Timber (CLT) has gained importance in modern timber construction. These engineered timber products are composed of cross-wisely glued wooden layers. The thick and orthogonal lay-up ensures dimensional stability and enhanced in-plane and out-of-plane mechanical properties allowing CLT usage as full-size prefabricated floors, walls or roofs. The prefabrication and the easy-assembling between panels yield a new modular and fast construction method with a bio-sourced material. A recent state-of-the-art report [2] highlighted the sharp increase of CLT production during last decades, as well as the possibility for this product to compete against the mineral-based construction materials.

Each CLT layer is made of lamellae placed side by side which can be glued or not on the narrow edge side. In the non-gluing case, lateral gaps are allowed up to $6 \mathrm{~mm}$ by the recent European standard requirements for CLT [3] (Figure 1a). Several studies pointed out the non-negligible influence of these gaps on the mechanical behavior $[4,5,6]$, especially when dealing with in-plane shear stress [7,8]. Enlarging the lateral gaps up to hundreds of millimeters leads to innovative lightweight and less expensive panels having a regular alternation of lamellae and voids (Figure 1b). When the voids are filled with insulating material, the thermal, fire and acoustical efficiency can be improved. The development of such innovative products is still limited, especially because of the lack of simplified methods for predicting their mechanical behavior.

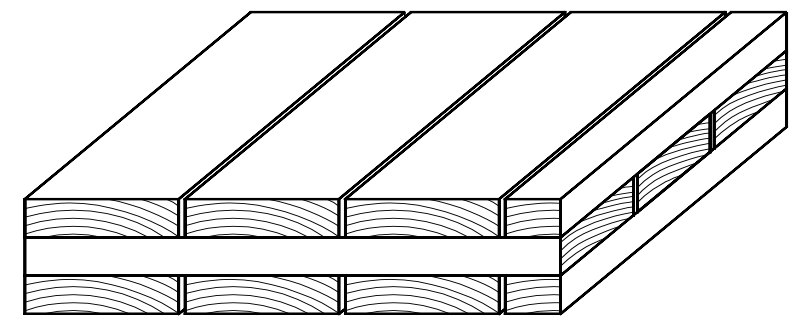

(a)

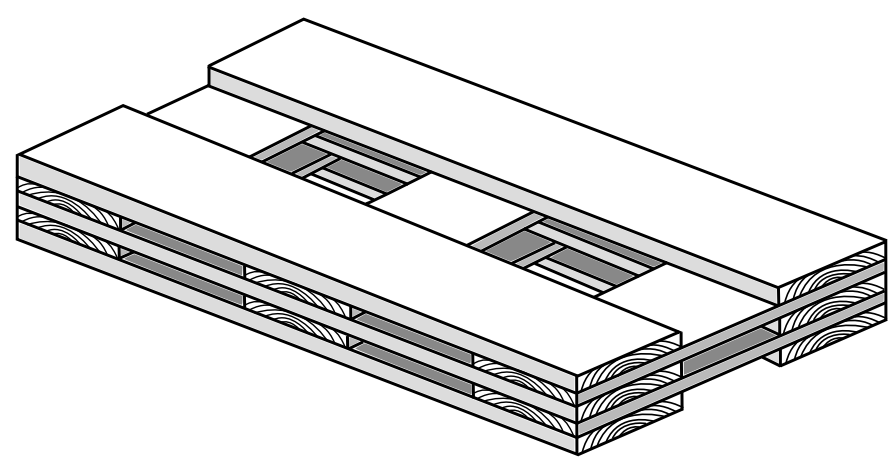

(b)

Figure 1: (a) CLT panel with lateral narrow gaps up to $6 \mathrm{~mm}$ as described in EN-16351 [3] and (b)innovative lightweight timber panels with wide gaps [1] 
To predict their mechanical behavior, CLT panels and lightweight timber products have been modeled as thick and periodic plates in a previous study by the authors [1]. The presence of the gaps has been taken into account by means of a plate homogenization scheme. The homogenization procedure links the strain energy of a unit-cell which is periodic within the panel with the strain energy of the whole panel, in order to estimate the homogenized plate stiffnesses. The plate membrane and bending stiffnesses were obtained imposing unit membrane and curvature strains to the unit-cell. To derive the plate shear force stiffness, the Bending-Gradient plate theory for thick plates has been applied [9, 10, 11, 12] by means of FE computations, which allow the estimation of the strain energy stored in the unit-cell and of the corresponding stress. The predicted bending and shear force stiffnesses, as well as maximum stresses at failure point, were in agreement with the experimental results of 4-points bending tests conducted by the authors [13, 1]. The relative difference between experiments and values predicted by FE homogenization were lower than $10 \%$ and most of the variability came from the experimental data. Moreover, the predicted variation of in-plane shear stiffness of CLT as a function of gaps was globally in agreement with test results of Brandner et al. [7] with a relative difference between experimental data lower than $15 \%$. In [1] existing closed-form approaches were also compared to FE homogenization and test results, showing that only the bending behavior can be predicted with such simplified approaches, while the homogenization procedure is needed to estimate the shear force stiffness, the in-plane shear and torsion stiffness. However, the need of the FE computation prevents the extension of the thick-plate modelling to practical cases.

In this paper, the thick-plate homogenization procedure is applied to timber panels having periodic gaps, in order to obtain closed-form expressions of stiffnesses and maximum stresses. The unit-cell is modeled as a space frame of beams connected each other by means of wooden blocks (Figure 2). Such space frame can be viewed as a periodic plate [14] and the simplified geometry of connected beams allows a closed-form estimation of the unit-cell strain energy. Indeed, the beam equations can be integrated since they depend only on one coordinate.

The paper is organized as follows: first, the timber panel with gaps is modeled as a space frame and the related notations are introduced in Section 2. In Section 3, the out-of-plane bending and in-plane shear stiffnesses are derived, while the shear force stiffness is derived within the subsequent Section 4. Then, Section 5 presents the derivation of closed-form solutions for predicting the maximum longitudinal and out-of-plane shear stress of CLT floors having gaps. Finally, the comparison between the obtained closed-form expressions, existing approaches and the reference FE results are showed in Section 6.

\section{CLT and timber panels with gaps as beam space frame}

CLT panels and lightweight timber products can be viewed as a 3D space frame of Timoshenko beams connected each other with wooden blocks (Figure 2). The blocks represent the glued parts of upper and lower lamellae, while the beams are the unglued/free parts of lamellae. The length of the beams being possibly zero, the use of Timoshenko model instead of Euler model is required.
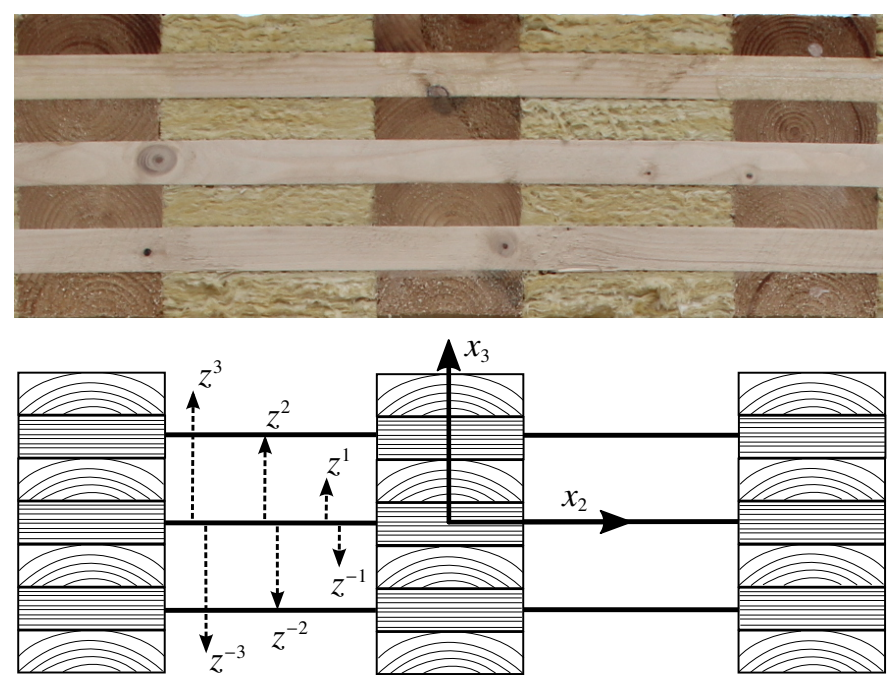

Figure 2: Cross section of timber panel with gaps modeled as beam space frame

The space frame of Figure 2 is a reproduction along the two in-plane directions of elementary unit-cells like Figure 3. Each unit-cell is made of $N$ beams connected each others with wooden blocks, where $N$ is the odd number of CLT layers. There are

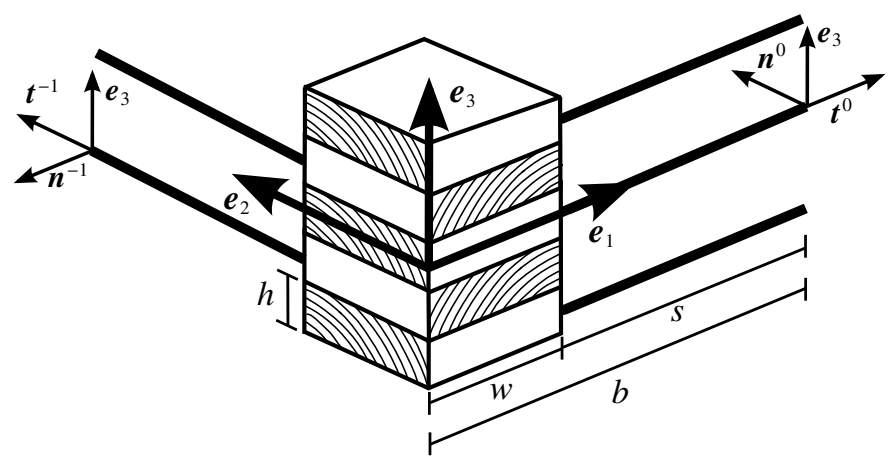

Figure 3: Periodic unit-cell of timber panel with gaps modeled as a space frame of beams connected with wooden blocks

$N_{l}$ longitudinal beams and $N_{c}$ cross beams, with $N=N_{l}+N_{c}$ and $N_{l}=\frac{N+1}{2}, N_{c}=\frac{N-1}{2}$. In this paper, $\mathcal{L}$ and $C$ stand for the sets of indices of the longitudinal and cross layers. The top and bottom beams are always oriented in the longitudinal direction $x_{1}$ (Figure 3). The unit-cell has all lamellae with the same thickness $h$ and width $w$, while the length of the unit-cell is $b$. The position of each beam with respect to the reference frame of Figure 2 is denoted $z^{i}=i h$, with $i \in\left[-\frac{N-1}{2} ; \frac{N-1}{2}\right]$. The mirror symmetry of the unit-cell with respect to the mid-plane $x_{3}=0$ ensures that $z^{i}=-z^{-i}$. Main notations are summarized in Table 1.

Each lamella is made of wood whose longitudinal direction coincides with the beam main axis. Since the annual rings orientation may not be known, wood is assumed to be transversely 
isotropic and the subscripts $(L, N, Z)$ of wood's moduli of elasticity stands for the material reference frame [13, 15, 16, 17]. The longitudinal direction is noted $L$, whereas the normal $N$ and out of plane $Z$ directions feature the same constitutive behavior $\left(E_{N}=E_{Z}\right.$ and $G_{L N}=G_{L Z}$ etc. $)$.

The $i^{\text {th }}$ beam local reference frame is $\left(\boldsymbol{t}^{i}, \boldsymbol{n}^{i}, \boldsymbol{e}_{3}\right)$ associated to the local coordinates $\left(y_{t}, y_{n}, x_{3}\right)$, and the elastic energy of a single beam can be evaluated as:

$$
U^{\text {beam }, i}=\int_{0}^{s} \frac{1}{2}\left(\boldsymbol{r}^{i} \cdot\left(\boldsymbol{F}^{i}\right)^{-1} \cdot \boldsymbol{r}^{i}+\boldsymbol{m}^{i} \cdot\left(\boldsymbol{H}^{i}\right)^{-1} \cdot \boldsymbol{m}^{i}\right) d y_{t}
$$

where $s$ is the length of the beam while $\boldsymbol{r}^{i}$ and $\boldsymbol{m}^{i}$ are respectively the resultant forces and moments that describe the beam stress state. The beam stiffness tensors $\boldsymbol{F}^{i}$ and $\boldsymbol{H}^{i}$ are:

$$
\boldsymbol{F}^{i}=\left(\begin{array}{ccc}
E_{L} S & 0 & 0 \\
0 & G_{L Z} S_{n} & 0 \\
0 & 0 & G_{L Z} S_{3}
\end{array}\right)_{\left(\boldsymbol{t}^{i}, \boldsymbol{n}^{i}, \boldsymbol{e}_{3}\right)}
$$

and

$$
\boldsymbol{H}^{i}=\left(\begin{array}{ccc}
G_{L Z} J & 0 & 0 \\
0 & E_{L} I_{n} & 0 \\
0 & 0 & E_{L} I_{3}
\end{array}\right)_{\left(\boldsymbol{t}^{i}, \boldsymbol{n}^{i}, \boldsymbol{e}_{3}\right)},
$$

where $E_{L}$ is the Young's modulus along longitudinal direction, $G_{L Z}$ the longitudinal shear modulus, $S=w h$ is the section area of the lamella, $S_{n}=S_{3}=\frac{5}{6} w h$ are the shear areas, $I_{n}=\frac{h^{3} w}{12}$ and $I_{3}=\frac{w^{3} h}{12}$ are the second moments of inertia and $J$ is the torsion constant defined as:

$$
J=\frac{w h^{3}}{16}\left(\frac{16}{3}-3.36 \frac{h}{w}\left(1-\frac{1}{12}\left(\frac{h}{w}\right)^{4}\right)\right)
$$

Following the approach of [14], the space frame of beams can be modeled as a periodic plate, and plate stiffnesses can be estimated by means of a closed-form homogenization procedure based on the prediction of beams' strain energy. In addition to the elastic energy of the beams, the elastic energy related to the deformation of wooden blocks must also be taken into account. As the following sections show, the wooden blocks have been modeled as rotational springs to predict the membrane and bending stiffnesses of the panel and as 3D blocks with a discrete kinematics to estimate the shear force stiffnesses.

\section{Membrane and bending stiffnesses of timber panel}

In this section, closed-form solutions for predicting the membrane and bending stiffnesses of CLT and innovative panels are derived. The wooden blocks are modeled as springs connecting upper and lower beams, deformable only under in-plane rotations and with a rotational stiffness $K_{\theta}$ (Figure 4). This is to take into account the torsion-like mechanism between upper and lower blocks that occurs when the panel is submitted to in-plane shear $[1,18]$. This simplified modeling follows the approach of several existing experimental and theoretical studies [19, 8]. It allows computing only the stress state of beams having full length $b$, while the strain energy of blocks is concentrated in the springs energy. $\alpha_{n}^{b} \quad$ In-plane shearing compliance

Eq. (14)

of lamellae

$\alpha_{n}^{*} \quad$ Corrected shearing compliance

of lamellae

$\alpha_{3}^{s} \quad$ Out-of-plane shearing compliance Eq. (40) of lamellae

$b \quad$ In-plane size of the unit cell $\quad$ Fig. 3

$C \quad$ Set of indices of cross lamellae $\quad$ Sec. 2

$\chi_{\alpha \beta} \quad$ Plate curvatures

$D_{i j} \quad$ Plate bending stiffness

$e_{\alpha \beta} \quad$ Plate membrane strains

$E_{L} \quad$ Wood parallel to the grain Young modulus

$G_{L Z} \quad$ Wood parallel to the grain shear modulus

$G_{Z N} \quad$ Wood rolling shear modulus

$G_{L Z}^{*} \quad$ Torsional reduced shear stiffness

$h \quad$ Lamella's thickness

$I_{n} \quad$ Out-of-plane $2^{\text {nd }}$ moment of

inertia of the lamella

$I_{3} \quad$ In-plane $2^{\text {nd }}$ moment of

inertia of the lamella

$J \quad$ Torsion constant of the lamella $\quad$ Eq. (4)

$K_{\theta} \quad$ Rotational stiffness Eq. (6)

$\mathcal{L} \quad$ Set of indices of longitudinal lamellae Sec. 2

$\boldsymbol{m}^{i} \quad$ Moment of lamella $i$

$M_{\alpha \beta} \quad$ Plate bending moment

$N \quad$ Number of plies

$N_{l} \quad$ Number of longitudinal plies

$N_{c} \quad$ Number of cross plies

$\varphi_{\alpha}^{i} \quad$ Horizontal slip of block $i$

$\psi_{\alpha} \quad$ Vertical slip of all blocks

$Q_{\alpha} \quad$ Plate shear force

$r^{i} \quad$ Resultant of lamella $i$

$s \quad$ Gap's width between lamellae

$S \quad$ Lamella's section

$S_{n} \quad$ In-plane shear area of the lamella

$S_{3} \quad$ Out-of-plane shear area of the lamella

$\boldsymbol{\theta}^{i} \quad$ Rotation of lamella $i$

$\boldsymbol{\theta}^{\text {ext }, i} \quad$ Applied relative rotation of lamella $i$

$\boldsymbol{u}^{i} \quad$ Displacement of lamella $i$

$\boldsymbol{u}^{\text {ext, } i}$ Applied relative displacement of lamella $i$

$\boldsymbol{U}^{i} \quad$ Displacement of block $i$

$w \quad$ Lamella's width

$x_{3}^{i} \quad$ Relative out-of-plane coordinate

$z_{i} \quad$ Out-of-plane distance of lamella $i$

Eq. (1)

Sec. 4

Sec. 2

Sec. 2

Sec. 2

Fig. 8

Fig. 8

Sec. 4

Eq. (1)

Fig. 3

Eq. (2)

Eq. (2)

Eq. (2)

Fig. 6

Fig. 6

Fig. 8

Fig. 3

Fig. 8

Fig. 2

Table 1: Notations

Rotational stiffness. The expression of the rotational stiffness $K_{\theta}$ has been found in the available literature. Indeed, the mechanical behavior of two orthogonally glued wooden lamellae under relative in-plane rotation has been object of several experimental and theoretical studies [20, 19]. In particular, Moosbrugger et al. [8] considered a reduction of the CLT in-plane 


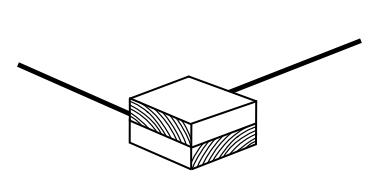

(a)

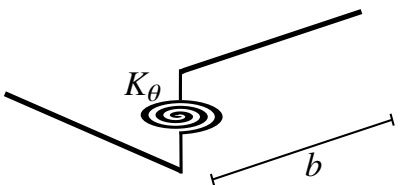

(b)
Figure 4: Wooden blocks connecting beams modeled as rotational spring deformable under in-plane rotation

shear stiffness $G_{L Z}^{*}$ by the presence of gaps independently from the number of layers and by a torsion-like mechanism of in-plane rotation of lamellae (Figure 5) with:

$$
G_{L Z}^{*}=\frac{G_{L Z}}{3 G_{\mathrm{eff}}}\left(\frac{w}{h}\right)^{2}
$$

where $G_{\text {eff }}=\frac{G_{L Z}+G_{Z N}}{2}$. Therefore, the reduction of in-plane

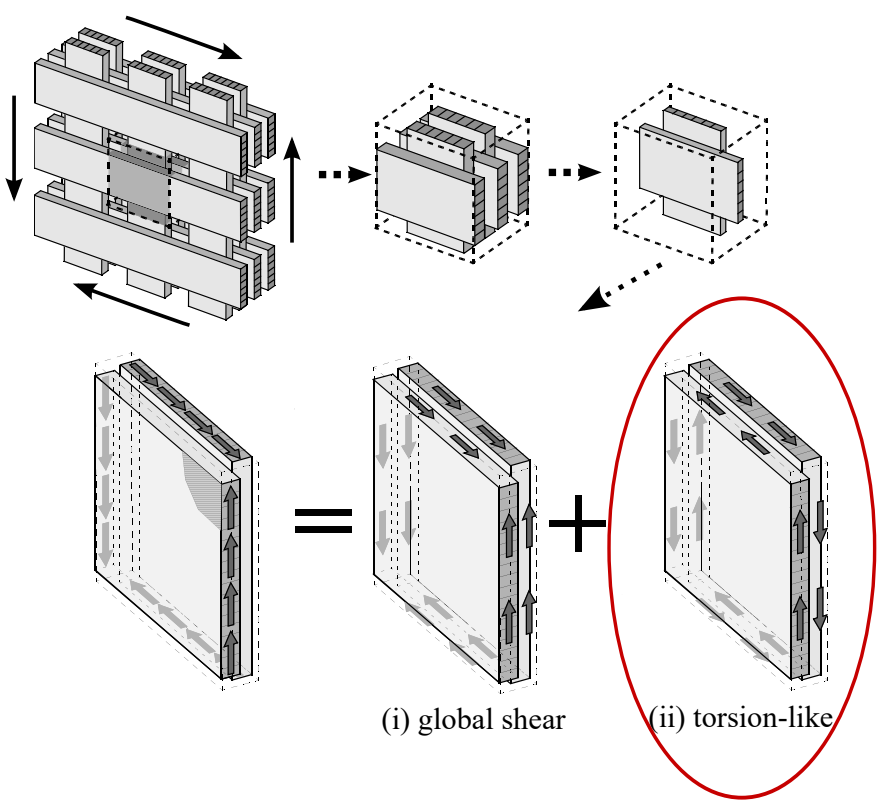

Figure 5: Torsion-like mechanism of glued lamellae in CLT from [8]

shear stiffness by the torsion-like mechanism is considered to be dependent on the aspect-ratio of lamellae $w / h$ but also on rolling shear modulus of wood, confirming the results of Jeitler [21]. In order to adapt this approach to our case, the rotational spring stiffness of the connection between two lamellae having volume $h w^{2}$ is:

$$
K_{\theta}=h w^{2} G_{L Z}^{*}=\frac{2 G_{L Z} w^{4}}{3 h\left(G_{L Z}+G_{Z N}\right)}
$$

Unit-cell problem. The macroscopic membrane and bending strains of the equivalent plate are imposed to the unit-cell by means of enforced relative beam displacements $\boldsymbol{u}^{\text {ext, } i}$ and rotations $\boldsymbol{\theta}^{\text {ext, } i}$, taking into account periodicity conditions. The applied displacements on beam extremities are equivalent to those applied in the 3D case on lateral faces of the unit-cell by Franzoni et al. [1]. When dealing with beams, the energy is also carried by beams' rotations [14]. Figure 6 shows the applied displacements and rotations as function of membrane $\boldsymbol{e}$ and curvature $\chi$ strains. $e_{11}$ and $e_{22}$ are axial membrane strains,

Axial membrane and out-of-plane bending loads

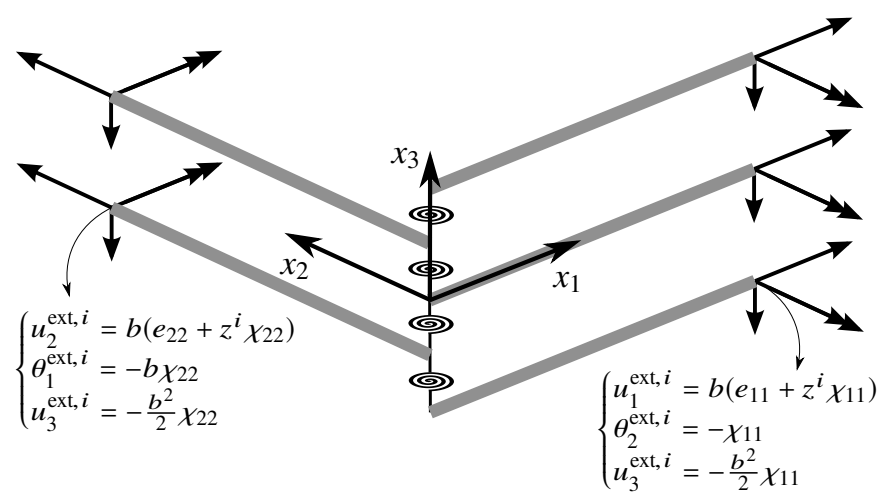

In-plane shear and torsion loads

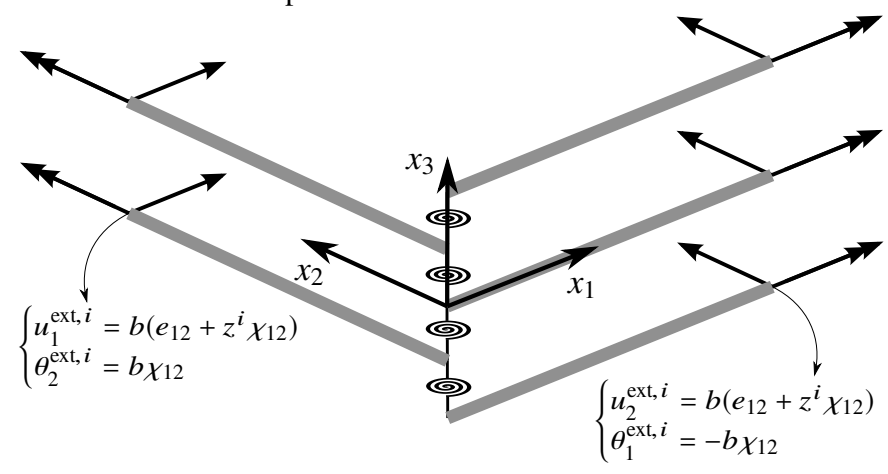

Figure 6: Membrane and curvature relative displacement of the equivalent plate applied to a 5-ply unit-cell of a panel (only non-vanishing prescribed displacements are detailed, cf Appendix A)

$\chi_{11}$ and $\chi_{22}$ are out-of-plane curvatures. These macroscopic strains are symmetric with respect to a plane of normal $\boldsymbol{e}_{1}$. The skew symmetric strains are the in-plane shear strain $e_{12}$ and the torsional curvature $\chi_{12}$. The detailed derivation of each beam stress state and the corresponding elastic energy is presented in Appendix A.

\subsection{Membrane, bending stresses and stiffnesses related to sym- metric generalized strains}

When the unit-cell is submitted to membrane strains $e_{11}$ and $e_{22}$ as well as curvatures strains $\chi_{11}$ and $\chi_{22}$, the resultants and moments of the beams are directly determined and the rotational springs are not loaded. The normal resultant forces $r_{t}^{i}$ and outof-plane moments $m_{n}^{i}$ of beams are derived from Equations A.9 and A.10 vanishing $e_{12}$ and $\chi_{12}$. They write in the local beam reference frame as:

$$
i \in \mathcal{L}:\left\{\begin{array}{l}
r_{t}^{i}=E_{L} S\left(e_{11}+z^{i} \chi_{11}\right) \\
m_{n}^{i}=E_{L} I_{n} \chi_{11}
\end{array}\right.
$$

and

$$
i \in C:\left\{\begin{array}{l}
r_{t}^{i}=E_{L} S\left(e_{22}+z^{i} \chi_{22}\right) \\
m_{n}^{i}=E_{L} I_{n} \chi_{22}
\end{array}\right.
$$


which are very similar to those found in [14]. The remaining components of forces and moments vanish.

Averaging the corresponding elastic energy over the unitcell surface leads to the following closed-form solution of outof-plane bending stiffness along $x_{1}$-direction $D_{11}$ :

$$
D_{11}=\frac{N_{l} E_{L} I_{n}}{b}+\frac{E_{L} S}{b} \sum_{i \in \mathcal{L}} z_{i}^{2}=\frac{E_{L} h^{3} w}{24 b}(N+1)\left(N^{2}+2 N-2\right)
$$

The result is classical: the bending stiffness $D_{11}$ is a contribution of the $N_{l}$ longitudinal beams bending stiffness $E_{L} I_{n}$ and the transport term related to the position of each longitudinal beam with respect to the mid-plane of the plate.

Similarly, the bending stiffness along $x_{2}$-direction $D_{22}$ as well as the membrane stiffnesses $A_{11}$ and $A_{22}$ are:

$$
\begin{gathered}
D_{22}=\frac{N_{c} E_{L} I_{n}}{b}+\frac{E_{L} S}{b} \sum_{i \in C} z_{i}^{2}=\frac{E_{L} h^{3} w}{24 b}(N-1)\left(N^{2}-2 N-2\right) \\
A_{11}=\frac{N_{l} E_{L} S}{b} \text { and } A_{22}=\frac{N_{c} E_{L} S}{b}
\end{gathered}
$$

Finally, the in-plane and out-of-plane Poisson coupling stiffnesses $A_{12}$ and $D_{12}$ vanish. This is a consequence of the modeling of the unit-cell with beams. Furthermore, in the FE study [1], it was already observed numerically that these couplings were extremely small and not reported in details.

\subsection{In-plane shear and torsional stiffness}

When the unit-cell is submitted to in-plane shear strain $e_{12}$ and torsion curvature $\chi_{12}$, the resultants and moments of the beams depend on the unknown in-plane rotations $\theta_{3}^{0, i}$ of beams at their connection with rotational springs $\left(y_{t}=0\right)$. They are derived from Equations A.9 and A.10 vanishing $e_{11}, e_{22}, \chi_{11}$ and $\chi_{22}$. The non-zero components of the resultants and moments write in the local beam reference frame as:

$$
i \in \mathcal{L}:\left\{\begin{array}{l}
r_{n}^{i}=\frac{b}{\alpha_{n}^{b}}\left[\left(e_{12}+z^{i} \chi_{12}\right)-\theta_{3}^{0, i}\right] \\
m_{t}^{i}=-G_{L Z} \chi_{12} \\
m_{3}^{i}=r_{n}^{i}\left(y_{t}-\frac{b}{2}\right)
\end{array}\right.
$$

and

$$
i \in C:\left\{\begin{array}{l}
r_{n}^{i}=\frac{b}{\alpha_{n}^{b}}\left[-\left(e_{12}+z^{i} \chi_{12}\right)-\theta_{3}^{0, i}\right] \\
m_{t}^{i}=-G_{L Z} \chi_{12} \\
m_{3}^{i}=r_{n}^{i}\left(y_{t}-\frac{b}{2}\right)
\end{array}\right.
$$

where:

$$
\alpha_{n}^{b}=\frac{b}{G_{L Z} S_{n}}+\frac{b^{3}}{12 E_{L} I_{3}}
$$

is the sum of beam bending and shear compliances. It is the apparent compliance of in-plane bending of beams under uniform shear force. Therefore the derivation of in-plane shear $A_{33}$ and torsional $D_{33}$ stiffnesses requires the determination of all rotations $\theta_{3}^{0, i}$ which load the rotational springs.

There are no external beam loadings but only imposed displacements and rotations, hence the unit-cell elastic energy is also the unit-cell potential energy. Taking into account the contribution of rotational springs, the total potential energy of the unit-cell for the membrane and curvature loadings write as:

$$
W^{\text {thin }}=\sum_{i=-\frac{N-1}{2}}^{\frac{N-1}{2}} U^{\text {beam }, i}\left(\theta_{3}^{0, i}\right)+\sum_{j=-\frac{N-1}{2}}^{\frac{N-3}{2}} \frac{1}{2} K_{\theta}\left(\theta_{3}^{0, j+1}-\theta_{3}^{0, j}\right)^{2}
$$

where $U^{\text {beam, } i}\left(\theta_{3}^{0, i}\right)$ is defined in Equation (A.16).

Minimizing the potential energy with respect to the unknown kinematic variables allows their explicit determination through a linear system of $N$ equations whose solution may not write in a compact form. Nevertheless, for a 3-ply there are only three variables and closed-form expressions of the in-plane shear $A_{33}$ and torsional $D_{33}$ stiffnesses can be simply found:

$$
A_{33}^{3-p l y}=\frac{4 K_{\theta}}{3 \alpha_{n}^{*} K_{\theta}+b^{2}}
$$

and

$$
D_{33}^{3 \text {-ply }}=\frac{3 G_{L Z} J}{2 b}+\frac{h^{2} K_{\theta}}{\alpha_{n}^{*} K_{\theta}+b^{2}} .
$$

where $\alpha_{n}^{*}$ is a corrected apparent stiffness which will be discussed below. Furthermore, simplified general expressions of in-plane shear and torsional stiffnesses can be derived as the sum of compliances of the two limit cases when the dominant regime is either the rotational spring regime or the slender beam regime. In other words, assuming either $K_{\theta} \gg 1 / \alpha_{n}$ or $K_{\theta} \ll 1 / \alpha_{n}$ in the potential energy (Equation (15)) means finding the limit cases when the interfaces between beams are clamped connections (wide gaps, Figure 7a) or when the beams are rigid and only the blocks contribute to the energy (narrow gaps, Figure 7b). Taking the sum of the compliances of the limit

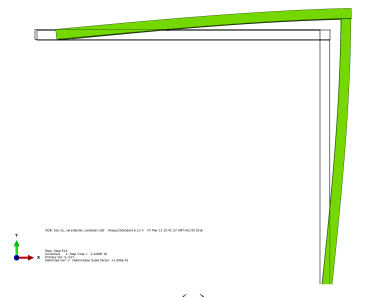

(a) (b)

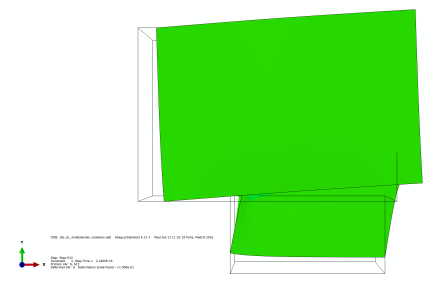

Figure 7: FE unit-cell under in-plane shear loading [1] for two limit cases: (a) slender beam regime at wide gaps $\left(K_{\theta} \gg 1 / \alpha_{n}\right)$ and (b) rotational spring regime at narrow gaps $\left(K_{\theta} \ll 1 / \alpha_{n}\right)$

cases leads to the following closed-form solutions for predicting the in-plane shear and torsional stiffnesses of timber panels with gaps:

$$
\begin{gathered}
A_{33}^{N-\text { ply }}=(N-1)\left(\frac{b^{2}}{2 K_{\theta}}+\frac{2 N \alpha_{n}^{*}}{N+1}\right)^{-1} \\
D_{33}^{N-\text { ply }}=\frac{N G_{L Z} J}{2 b}+\frac{N h^{2}(N-1)}{6}\left(\frac{b^{2}}{K_{\theta}(N-2)}+\frac{4 \alpha_{n}^{*}}{N+1}\right)^{-1}
\end{gathered}
$$


Further analyses not reported in this paper pointed out that the relative error of the simplified closed-form solutions (18) and (19) compared to the exact closed-form solutions has a maximum value of $8 \%$ and in most cases below $3 \%$.

The modeled rotational spring takes into account the inplane rotation mechanism between upper and lower blocks but not the gross in-plane shear of blocks. This contribution can be taken into account introducing a correction factor on the beam length $b$. Indeed, when $K_{\theta}=\infty$ and $b=w$, Equations (18) and (19) should return the in-plane shear and torsional stiffness of a massive CLT panel having glued lateral lamellae. This is possible when introducing the corrected apparent beam compliance:

$$
\alpha_{n}^{*}=\frac{24 b-19 w}{20 G_{L Z} w h}+\frac{(b-w)^{3}}{E_{L} w^{3} h} .
$$

The derivation of the correction of the beam compliance $\alpha_{n}^{*}$ is detailed in Appendix B.

\section{Shear force stiffnesses of timber panel}

It was demonstrated that for periodic plates in general, the relevant equivalent thick plate model is not the classical Reissner-Mindlin model but its generalization, the BendingGradient plate model [9, 14, 12]. This more comprehensive model was applied to timber panels with gaps in [1] by means of finite elements computations. In this specific case, it appeared that it is possible to derive a Reissner-Mindlin model with a reasonable approximation, except for 3-ply innovative panels with wide gaps. This is done, assuming that the plate is under uni-axial bending in each of its main directions. In such case, the shear forces $Q_{1}$ and $Q_{2}$ derive as follows from the bending moment: $Q_{1}=M_{11,1}, Q_{2}=M_{22,2}$. Since the plate is orthotropic, the Reissner-Mindlin shear force compliance $f_{11}^{\mathrm{RM}}$ is associated to $Q_{1}$ shear force occurring in the plane of normal $\boldsymbol{e}_{1}$, while the shear force compliance $f_{22}^{\mathrm{RM}}$ is related to $Q_{2}$ occurring in the plane of normal $\boldsymbol{e}_{2}$ and the coupling modulus $f_{12}^{\mathrm{RM}}$ vanish.

In order to derive a closed-form solution, the complete unitcell of Figure 3 is considered. This requires imposing a shear kinematics to the whole blocks and deriving their contribution to the elastic energy. More precisely, the kinematics of each block will be described by a finite number of discrete degrees of freedom and be made compatible with the one of adjacent blocks as well as the kinematics of the connected beams. Once the kinematics of the unit-cell is defined, the loading corresponding to shear forces is introduced. Then, the stress state of the unit-cell under shear forces as well as the Reissner-Mindlin compliance moduli are presented.

\subsection{Kinematics of the unit-cell}

\subsubsection{Out-of-plane shear kinematics of wooden blocks and re- lated energy}

An out-of-plane shear kinematics is imposed to blocks constituting the thick interface between a longitudinal lamella and a cross lamella. An example is presented in Figure 8 for the "1-3" shear direction (plane $x_{2}=0$ ), where the upper-block is a half cross lamella and the lower-block is a half longitudinal lamella.

The shear kinematics is a superposition of horizontal and vertical slips of wooden blocks. The displacement field associated to such kinematics showed in Figure 8 writes for the longitudinal block as:

$$
\boldsymbol{u}=\left\{\begin{array}{l}
u_{1}=U_{1}^{i}+x_{3}^{i} \varphi_{2}^{i}, \\
u_{2}=0 \\
u_{3}=U_{3}^{i}-\psi_{2} x_{1},
\end{array}\right.
$$

and for the cross block:

$$
\boldsymbol{u}=\left\{\begin{array}{l}
u_{1}=U_{1}^{i+1}+x_{3}^{i+1} \phi^{i, i+1}, \\
u_{2}=0, \\
u_{3}=U_{3}^{i+1}-\psi_{2} x_{1},
\end{array}\right.
$$

where $\boldsymbol{U}^{i}$ are the displacements of the blocks at $x_{1}=x_{2}=0$ and $x_{3}=z^{i}$ and $x_{3}^{i}=x_{3}-z^{i}$ is the local out-of-plane coordinate. The slip coefficient $\phi_{2}^{i, i+1}$ in Figure 8 is to be determined and $\psi_{2}$ is a slip coefficient representing the inclination of all blocks.

Imposing the continuity of vertical displacements $u_{3}$ at the interface enforces $U_{3}^{i}=U_{3}^{i+1}$, meaning that a relative out-ofplane displacement between blocks is not permitted. Imposing the continuity of horizontal displacement field $u_{1}$ at blocks interface, sets the unknown slip coefficient $\phi_{2}^{i, i+1}$ :

$$
\frac{h}{2} \varphi_{2}^{i}+U_{1}^{i}=\frac{h}{2} \phi_{2}^{i, i+1}+U_{1}^{i+1} .
$$

Now the out-of-plane shear strain $\varepsilon_{13}^{l}$ of the longitudinal block can be evaluated as:

$$
2 \varepsilon_{13}^{l}=\varphi_{2}^{i}-\psi_{2}
$$

and for the cross block:

$$
2 \varepsilon_{13}^{c}=\phi_{2}^{i, i+1}-\psi_{2}=\frac{2}{h}\left(U_{1}^{i+1}-U_{1}^{i}\right)-\psi_{2}-\varphi_{2}^{i}
$$

These formulas remain unchanged for an interface where the lower block is a cross lamella and the upper block is a longitudinal lamella provided the following definition for the rotation of cross blocks is introduced:

$$
\varphi_{2}^{i} \equiv \psi_{2} \quad \text { for } \quad i \in C,
$$

where it becomes implicit that all rotations $\varphi_{2}^{i}$ of cross blocks are identical.

Once the out-of-plane shear strains along shear direction " 1 3" are estimated, the interface elastic energy can be evaluated as the sum of half volume strain energy of longitudinal and cross blocks:

$$
W^{(i, i+1), \varepsilon_{13}}=\frac{1}{2} \frac{h w^{2}}{2}\left[G_{L Z}\left(2 \varepsilon_{13}^{l}\right)^{2}+G_{Z N}\left(2 \varepsilon_{13}^{c}\right)^{2}\right]
$$

which becomes, substituting the shear strains in Equations (25) and (24):

$$
\begin{aligned}
W^{(i, i+1), \varepsilon_{13}=}= & \frac{1}{2} \frac{h w^{2}}{2}\left[G_{L Z}\left(\varphi_{2}^{i+1}-\varphi_{2}^{i}\right)^{2}\right. \\
& \left.+G_{Z N}\left(\frac{2}{h}\left(U_{1}^{i+1}-U_{1}^{i}\right)-\varphi_{2}^{i+1}-\varphi_{2}^{i}\right)^{2}\right]
\end{aligned}
$$




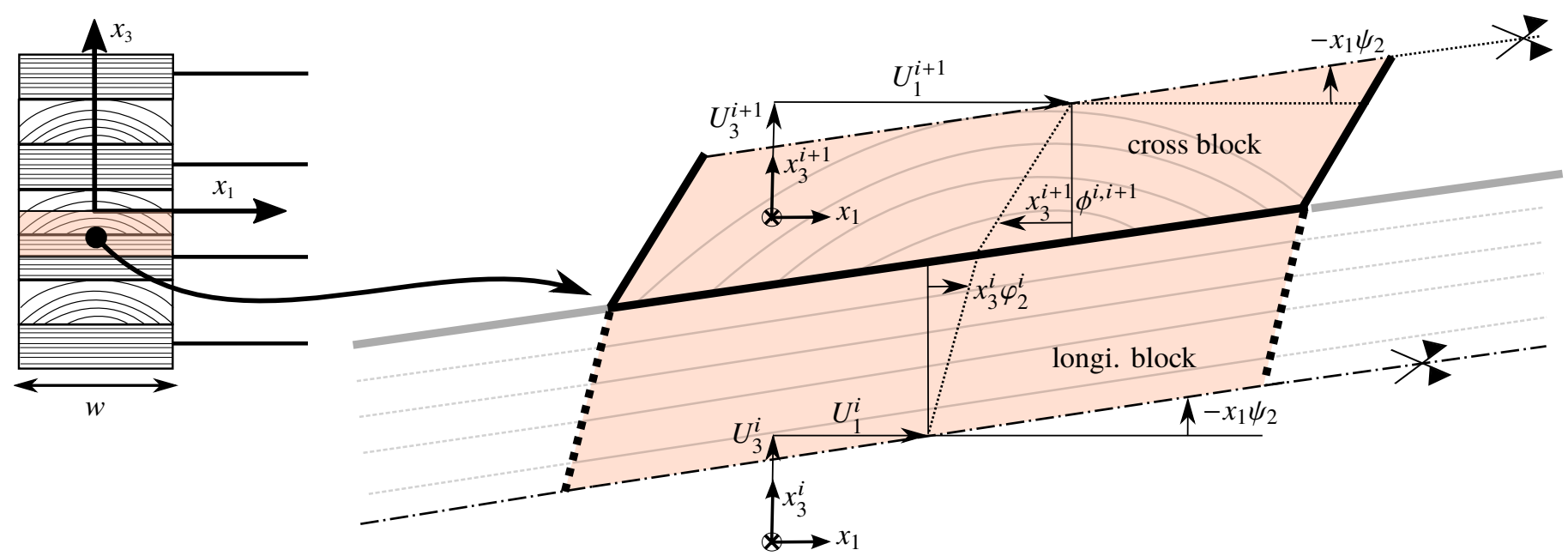

Figure 8: Out-of-plane shear kinematics in plane $x_{2}=0$ of the thick interface between lamellae $\left(\psi_{2}<0\right)$

The same derivation of out-of-plane shear strain and associated energy can be performed for the shear direction " $2-3$ ", leading to:

$$
\begin{aligned}
W^{(i, i+1), \varepsilon_{23}=}= & \frac{1}{2} \frac{h w^{2}}{2}\left[G_{L Z}\left(\varphi_{1}^{i+1}-\varphi_{1}^{i}\right)^{2}\right. \\
& \left.+G_{Z N}\left(\frac{2}{h}\left(U_{2}^{i+1}-U_{2}^{i}\right)+\varphi_{1}^{i+1}+\varphi_{1}^{i}\right)^{2}\right]
\end{aligned}
$$

The orthotropy symmetry of CLT with gaps ensures the uncoupling between out-of-plane shear effects occurring in the $x_{2}=0$ plane and in the $x_{1}=0$ plane, and hence it is possible to sum the shear strain energies derived for each shear plane.

Note that the contributions of out-of-plane shear strain $\varepsilon_{13}^{l}$ in the longitudinal blocks at top and bottom free faces of the unitcell are considered to be null, since they involve free edges that do not carry much strain energy. Even if this hypothesis is not strictly kinematically compatible, it is a better approximation which is also made in the shear analogy method [22].

\subsubsection{Beam kinematics and periodicity conditions}

In Section 3, blocks were reduced to a rotational spring. Here, because of the in-plane size of the blocks, the total length of the beams is $s=b-w$. With Figure 8 as reference, the displacement $\boldsymbol{u}$ of the longitudinal block must be continuous at $x_{1}= \pm w / 2$ with the kinematics of the longitudinal beam $\boldsymbol{u}^{i}$. For instance, this enforces for the out-of-plane displacement $u_{3}^{i}$ :

$$
u_{3}^{0, i}=U_{3}^{i}+\frac{w}{2} \psi_{2} \quad \text { and } \quad u_{3}^{s, i}=U_{3}^{i}-\frac{w}{2} \psi_{2}
$$

where $u_{j}^{0, i}=u_{j}^{i}\left(y_{t}=0\right)$ is the displacement of beam $i$ at its first extremity and $u_{j}^{s, i}=u_{j}^{i}\left(y_{t}=s\right)$ is the displacement at its final extremity. Similar arguments leads to the following periodicity contraints between the end displacements of the beams:

$$
\boldsymbol{u}^{s, i}-\boldsymbol{u}^{0, i}=w \boldsymbol{t}^{i} \times \boldsymbol{\psi} \quad \text { with } \quad \boldsymbol{\psi}=\left(\begin{array}{c}
\psi_{1} \\
\psi_{2} \\
0
\end{array}\right)
$$

In order to ensure rotation continuity between blocks and beams, the rotation of each block $\varphi^{i}$ must equal the beam's rotation at both extremities:

$$
\theta^{0, i}=\varphi^{i} \text { and } \boldsymbol{\theta}^{s, i}=\varphi^{i}
$$

leading to the following periodicity constraints on the end rotations of the beams:

$$
\boldsymbol{\theta}^{0, i}-\boldsymbol{\theta}^{s, i}=\mathbf{0}
$$

\subsection{The unit-cell loading}

The periodic homogenization procedure considers, in the $3 \mathrm{D}$ continuum case, the stress in equilibrium with a body force deriving from the variations of the bending moment [9]. According to Lebée and Sab [14], in the case of beam space frame, this body force becomes beam distributed forces $\boldsymbol{q}^{i}$ and torques $\boldsymbol{l}^{i}$. These loads derive from the stress state associated to the curvatures $\chi$ found in Equation (7). The following beam distributed forces $\boldsymbol{q}^{i}$ for longitudinal and cross beams can be found as a function of the shear forces $Q_{1}$ and $Q_{2}$ :

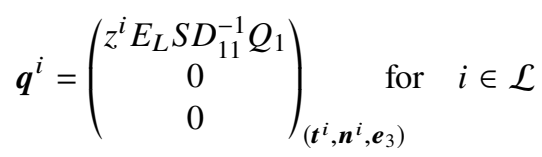

and

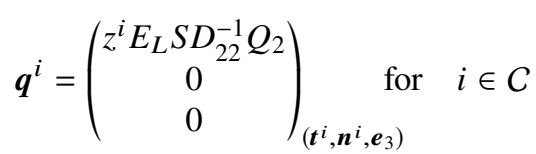

Moreover, the distributed torques $\boldsymbol{l}^{i}$ applied on each beam are derived as: $\boldsymbol{l}^{i}=\boldsymbol{l}^{s / 2, i}+\boldsymbol{t}^{i} \times \boldsymbol{q}^{i}\left(y_{t}-s / 2\right)$, where:

$$
\boldsymbol{l}^{s / 2, i}=\left(\begin{array}{c}
0 \\
E_{L} I_{n} D_{11}^{-1} Q_{1} \\
0
\end{array}\right)_{\left(\boldsymbol{t}^{i}, \boldsymbol{n}^{i}, \boldsymbol{e}_{3}\right)}^{\text {for }} \quad i \in \mathcal{L}
$$

and

$$
\boldsymbol{l}^{s / 2, i}=\left(\begin{array}{c}
0 \\
E_{L} I_{n} D_{22}^{-1} Q_{2} \\
0
\end{array}\right)_{\left(\boldsymbol{t}^{i}, \boldsymbol{n}^{i}, \boldsymbol{e}_{3}\right)}^{\text {for }} \quad i \in C
$$




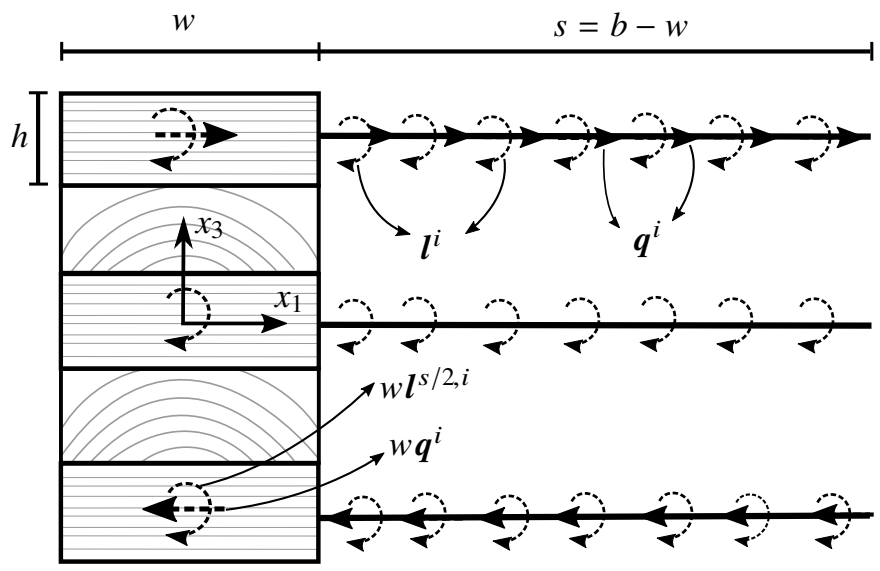

Figure 9: Unit loads for the thick space frame analysis on a 5-ply unit-cell

Figure 9 shows the applied loads to a unit-cell of a panel. The wooden blocks are also submitted to concentrated forces $w \boldsymbol{q}^{i}$ and torques $w \boldsymbol{l}^{s / 2, i}$, obtained integrating expressions (34) and (36) over the length $w$ of blocks.

The action of the loadings $\boldsymbol{q}^{i}$ and $\boldsymbol{l}^{i}$ is analogous to Jouravski formula for the shear stress in thin-walled beams [23] and may be globally understood as follows. The longitudinal load $q_{t}^{i}$ generates a resultant force in the longitudinal direction applied by each beam to its corresponding block. This resultant being different for each beam, the cross block between two longitudinal beam is submitted to a horizontal shearing force.

\subsection{Stress state in the unit-cell and shear force compliance}

The procedure for determining the stress state of beams and the related elastic energy when the unit-cell is submitted to shear forces is very similar to the procedure introduced in Section 3 and detailed in Appendix A for membrane and curvature loadings. The only differences are the presence of external beam loadings $\boldsymbol{q}^{i}$ and $\boldsymbol{l}^{i}$, the modified periodicity conditions (Equations (31) and (33)) and fewer symmetry conditions. The stress state of each beam depends on the out-of-plane beam rotations $\theta_{1}^{0, i}$ and $\theta_{2}^{0, i}$ as well as the global inclinations of blocks $\psi_{\alpha}$ :

$$
i \in \mathcal{L}:\left\{\begin{array}{l}
r_{t}^{i}=z^{i} E_{L} S D_{11}^{-1} Q_{1}\left(y_{t}-\frac{s}{2}\right) \\
r_{3}^{i}=\frac{1}{\alpha_{3}^{s}}\left(w \psi_{2}+s \theta_{2}^{0, i}+Q_{1} \frac{s^{3}}{12 D_{11}}\right) \\
m_{n}^{i}=-\left(r_{3}^{i}+D_{11}^{-1} E_{L} I_{n} Q_{1}\right)\left(y_{t}-\frac{s}{2}\right)
\end{array}\right.
$$

and

$$
i \in C:\left\{\begin{array}{l}
r_{t}^{i}=z^{i} E_{L} S D_{22}^{-1} Q_{2}\left(y_{t}-\frac{s}{2}\right) \\
r_{3}^{i}=\frac{1}{\alpha_{3}^{s}}\left(-w \psi_{1}-s \theta_{1}^{0, i}+Q_{2} \frac{s^{3}}{12 D_{22}}\right) \\
m_{n}^{i}=-\left(r_{3}^{i}+D_{22}^{-1} E_{L} I_{n} Q_{2}\right)\left(y_{t}-\frac{s}{2}\right)
\end{array}\right.
$$

where:

$$
\alpha_{3}^{s}=\frac{s}{G_{L Z} S_{3}}+\frac{s^{3}}{12 E_{L} I_{n}},
$$

and the remaining terms of the stress state vanish.
Since the obtained shear strain energy of blocks is a function of out-of-plane rotations $\theta_{\alpha}^{0, i}$, global inclinations $\psi_{\alpha}$ and inplane displacements $U_{\alpha}^{i}$ (Equations (28) and (29)), the closedform expression of the unit-cell strain energy under the shear forces depends on the set of $3 N+2$ kinematic variables $\left(\theta_{1}^{i}\right.$ for $i \in C, \theta_{2}^{i}$ for $\left.i \in \mathcal{L}, U_{1}^{i}, U_{2}^{i}, \psi_{1}, \psi_{2}\right)$. As in Section 3.2, the unknown variables can be determined minimizing the potential energy stored in the unit-cell. Minimizing first with respect to the displacements $U_{\alpha}^{i}$ and setting $U_{\alpha}^{0}=0$ for avoiding a rigid translation of the unit-cell, leads to the direct determination of $2 N$ in-plane displacements [24]. The solution of the remaining linear system of $N+2$ equations may not write in a compact form. Hence, in order to simplify the expression of the strain energy, the rotations of beams of the same type (longitudinal or cross) are considered to be equal, leading to only two beam rotations and two inclinations within a unit-cell: $\theta_{1}^{l}, \theta_{2}^{c}, \psi_{1}$ and $\psi_{2}$. FE analyses revealed that this hypothesis is a reasonable approximation of the actual complex behavior of the unit-cell under out-of-plane shear strains. Then, the minimization with respect to the four unknown rotations finally yields the closedform expression of all kinematic variables and therefore of shear force stiffnesses.

The following expression of $f_{11}^{\mathrm{RM}}$ is obtained from the elastic energy:

$$
\begin{aligned}
& f_{11}^{\mathrm{RM}}=\frac{2 \alpha_{3}^{s}}{N+1}+\frac{b^{2}\left(N^{2}+2 N-3\right)}{2 h w^{2}\left(N^{2}+2 N-2\right)^{2}} \times \\
& {\left[\frac{\left[(b-2 w)\left(N^{2}+2 N-2\right)-b\right]^{2}}{b^{2} G_{L Z}(N-1)^{2}(N+3)}+\frac{6}{5 G_{Z N}} \frac{N^{2}+2 N+5}{N+1}\right]}
\end{aligned}
$$

The first term is related to the bending ans shear flexibility of beams, the second is associated to the longitudinal shear of blocks $G_{L Z}$ while the third term is the rolling shear compliance $G_{Z N}$ of blocks. Similarly, the expression of the shear force compliance $f_{22}^{\mathrm{RM}}$ related to shear force $Q_{2}$ is:

$$
\begin{aligned}
& f_{22}^{\mathrm{RM}}=\frac{2 \alpha_{3}^{s}}{N-1}+\frac{b^{2}\left(N^{2}-2 N-3\right)}{2 h w^{2}\left(N^{2}-2 N-2\right)^{2}} \times \\
& {\left[\frac{\left[(b-2 w)\left(N^{2}-2 N-2\right)-b\right]^{2}}{b^{2} G_{L Z}\left(N^{2}-1\right)(N-3)}+\frac{6}{5 G_{Z N}} \frac{N^{2}-2 N+5}{N-1}\right]}
\end{aligned}
$$

When $b=w$ and the CLT has no gaps, the first term $\frac{2 \alpha_{3}^{s}}{N+1}$ related to beams vanishes and Equations (41) and (42) return the shear force compliances of a CLT having glued narrow edges.

\section{Maximum longitudinal and rolling shear stress}

When dealing with the out-of-plane behavior of CLT and innovative timber panels, the governing failure modes are longitudinal tensile failures of bottom layer and rolling shear failure of cross layer. This was observed during many experimental investigation of the literature $[25,26,4,27,13]$. In the previous study by the authors [1], the tensile and rolling shear stresses at 
failure point of tested timber floors with wide gaps in 4-points bending have been predicted with the FE homogenization and were in agreement with mean strength values from the literature. In this Section, closed-form solutions for estimating the maximum longitudinal and rolling shear stresses are derived.

\subsection{Maximum longitudinal stress}

The maximum stress related to bending in direction $x_{1}$ occurs in the top and bottom longitudinal lamellae. Equation (7) gives the stress state of beams as function of the membrane strain and plate curvature. From the bending constitutive equation, the curvature may be written as function of the plate bending moment as: $\chi_{11}=\frac{M_{11}}{D_{11}}$. Hence the non-vanishing components of the resultant and moment in the top and bottom beams write as function of the plate bending moment as $M_{11}$ as:

$$
r_{t}^{ \pm \frac{N-1}{2}}= \pm \frac{N-1}{2} E_{L} h S \frac{M_{11}}{D_{11}} \quad \text { and } \quad m_{n}^{ \pm \frac{N-1}{2}}=E_{L} I_{n} \frac{M_{11}}{D_{11}}
$$

Finally, the longitudinal stress in the top and bottom beam may be recovered from the traction resultant and bending moment from Navier equation:

$$
\sigma_{11}=\frac{r_{t}^{ \pm \frac{N-1}{2}}}{S}+x_{3}^{ \pm \frac{N-1}{2}} \frac{m_{n}^{ \pm \frac{N-1}{2}}}{I_{n}}
$$

Combining these equations leads to the following expression of extremal longitudinal stress :

$$
\sigma_{11}^{\text {extr. }}= \pm \frac{12 b N M_{11}}{w h^{2}(N+1)\left(N^{2}+2 N-2\right)}
$$

Note that the plate bending moment $M_{11}$ is per unit length and therefore is normalized over the panel's width.

\subsection{Maximum rolling shear stress}

The mean rolling shear stress in cross blocks can be estimated as $\sigma_{13}^{i}=\frac{V^{i}}{w^{2}}$, where $V^{i}$ is the shearing force in direction $x_{1}$ on the considered wooden block. This force can be obtained from the equilibrium of forces showed in Figure 10. The plate

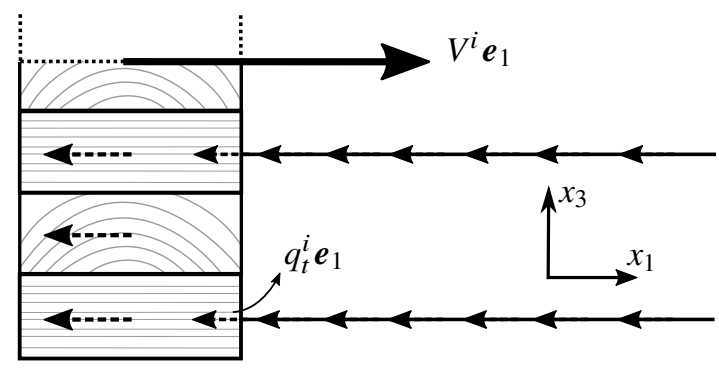

Figure 10: Shearing force $V^{i}$ from the equilibrium along $x_{1}$ direction

shear force loadings $q_{t}^{i}$ are axial distributed forces per unitlength and their resultant applied on the longitudinal block has to be in equilibrium with the shearing force $V^{i}$. Therefore substituting the closed-form expression of $q_{t}^{i}$ from Equation (34) leads to:

$$
V^{i}=\frac{w h b E_{L} Q_{1}}{D_{11}} \sum_{\substack{j \leq i-1 \\ j \in \mathcal{L}}} z^{j}
$$

where the partial sum operates only on longitudinal beams. The maximum shearing force is obtained when $i$ represents the position of the cross block closest to the mid-plane of the cross section. The corresponding average rolling shear stress in the most sheared cross block writes as:

$$
\sigma_{13}^{\text {extr. }}=\frac{24\left\lfloor\frac{N+2}{4}\right\rfloor^{2} b^{2} Q_{1}}{h w^{2}(N+1)\left(N^{2}+2 N-2\right)}
$$

where $\lfloor x\rfloor$ denotes the integer value of $x$.

\section{Comparison}

In this Section, the elastic behavior predicted by closed-form solutions is compared with the reference behavior predicted by the numerical homogenization [1] and existing simplified approaches. The derived closed-form expression for the bending stiffness is similar to the volume fraction approach already presented in [1]. This leads to the same conclusions which are not repeated here. The focus is given first on the in-plane shear and the torsion stiffness. Finally, the shear force stiffness as well as the extremal stress predictions are presented.

Regarding the plate stiffnesses, the results of each method are normalized with the stiffness of a CLT panel with glued narrow edges predicted by the FE method, in order to show the reduction of stiffness as a function of gaps.

\subsection{In-plane shear and torsion stiffness}

The comparison between Equations (18) and (19) predicting the in-plane shear and torsional stiffness and FE homogenization results from [1] is plotted in Figure 11 and 12 for different panel's lay-ups. The current approach from the working draft of CLT design section in the revised Eurocode 5 1-1 [28] and the effective modulus suggested by [8] are compared as well.

The simplified closed-form solution (18) for in-plane shear stiffness returns a very good agreement with the reference numerical results. Since Moosbrugger et al. [8] based their approach on a representative CLT sub-element having an infinite number of layers, such approach overestimates the in-plane shear stiffness for 3-ply and 5-ply, while is more in agreement with the 7-ply results.

Dealing with the torsional stiffness (19), the derived closedform expression are in good agreement with the reference behavior for timber panels with wide gaps made of lamellae having a small aspect ratio. When the the aspect ratio is large, the predicted torsional stiffness with all closed-form expressions deviates from the numerical results. For narrow gaps the overestimation of the stiffness remains acceptable, while for wide gaps the torsional stiffness is significantly underestimated. A possible explanation of this underestimation could be that the torsional warping of thin lamellae is prevented when they are glued together, leading to a higher global torsional stiffness than expected. Indeed, the closed-form approach assumes free torsion warping of lamellae.

The current approach of the working draft on CLT design of the new version of Eurocode 5 1-1 is based on the approach from Moosbrugger et al. [8], introducing FE fitting parameters to 

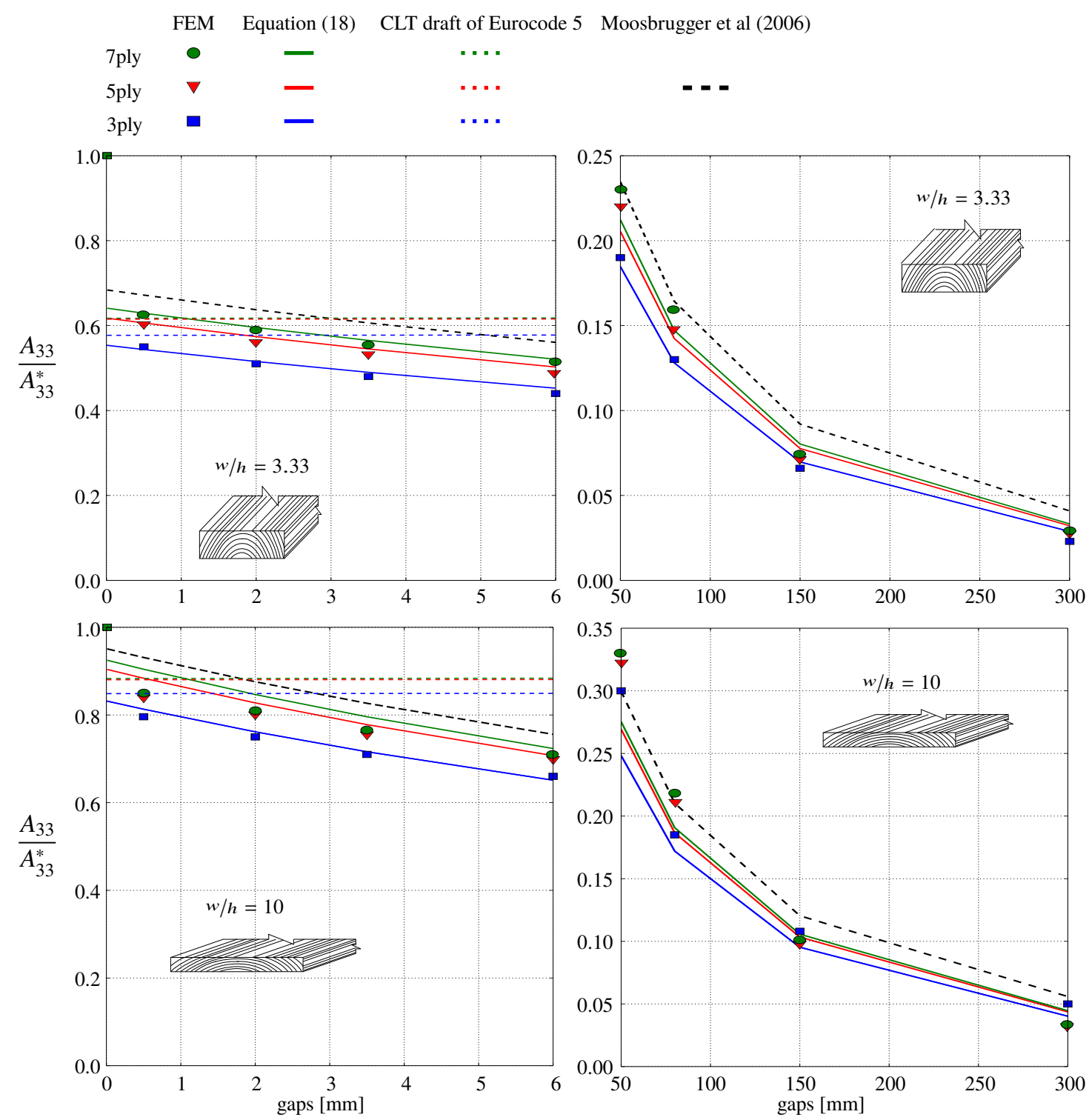

Figure 11: Comparison between closed-form solution, FE results and existing approaches for the in-plane shear stiffness $A_{33}$ for $w / h=3.33$ (top) and $w / h=10$ (bottom) 

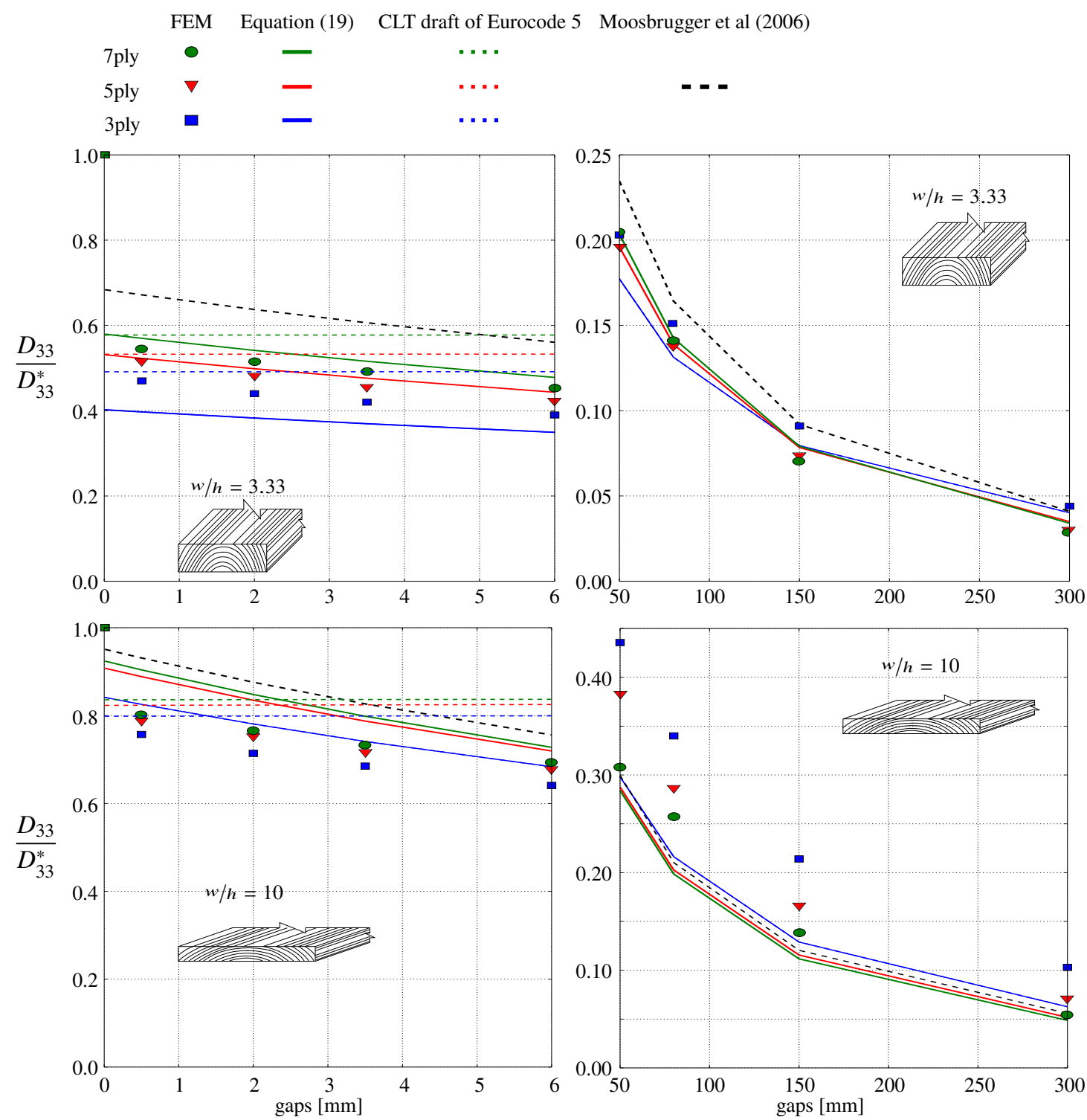

Figure 12: Comparison between closed-form solution, FE results and existing approaches for the torsional stiffness $D_{33}$ for $w / h=3.33$ (top) and $w / h=10$ (bottom) 

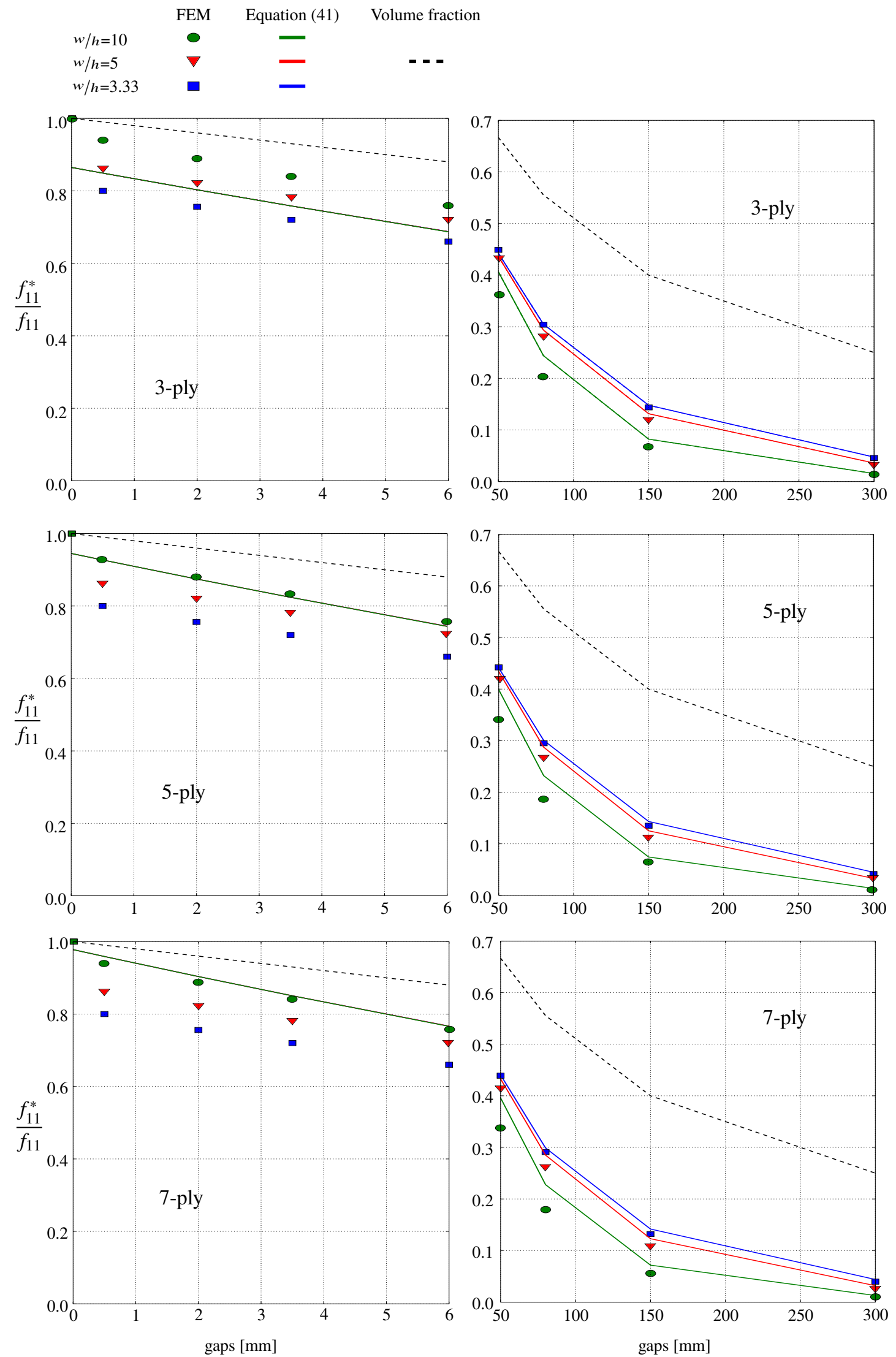

Figure 13: Comparison between closed-form solution, homogenization results and volume fraction approach for the shear force stiffness. 3-ply (top), 5-ply (middle) and 7-ply (bottom) 
take into account a finite number of layers [18, 29]. This method gives a single value of reduced stiffnesses due to unglued lateral lamellae, neglecting the influence of gaps up to $6 \mathrm{~mm}$. As Figure 11 and 12 show, the narrow gaps have a non-negligible influence of the reduction of CLT in-plane stiffnesses and may lead to a $20 \%$ drop of the predicted stiffness. In practice, this drop may be not so severe since, $6 \mathrm{~mm}$ is the maximum admitted length of gaps by [3] and does not reflect the average spacing. Another mitigating effect is the filling of gaps with glue during the pressing of layers [7], increasing so the in-plane rigidity of the panel. The closed-form solutions presented here have the advantage of being conservative and extended to innovative panels with wide gaps.

\subsection{Shear force stiffness}

In Figure 13, the reduction of the shear force stiffness predicted with the closed-form expression (41) and the numerical homogenization results are compared for different lay-ups.

At narrow gaps, all closed-form solutions are almost superimposed showing no dependency from the lamella's aspect ratio $w / h$, while the numerical homogenization shows dependency. This is because the out-of-plane shear of lamellae having lateral free edges leads to additional stresses perpendicular to the grain which are dependent on the lamella's aspect ratio. These effects are well predicted by the FEM modeling but they are not taken into account in the present closed-form approach. Introducing a modified rolling shear modulus depending on the lamellae aspect ratio would improve the prediction.

Furthermore, while increasing the number of layers, progressive overestimation of the shear force stiffness is observed, especially at narrow gaps. This derives from the kinematic hypothesis of equal rotations of blocks having the same orientation made in Section 4.3. For the 3-ply lay-up such hypothesis is trivially satisfied. However, the more the number of layers increases, the more this hypothesis deviates from the actual kinematics. For 5-ply and 7-ply, the closed-form expression still returns an acceptable approximation of the reference behavior.

Globally, the derived closed-form solution for predicting the shear force compliance of CLT and innovative panels are in good agreement with the reference numerical results at both narrow and wide gaps. On the contrary, the simplified approach of volume fraction strongly overestimates the shear force stiffness, especially at wide gaps.

\subsection{Maximum longitudinal and rolling shear stresses}

The suggested closed-form expressions (45) and (47) for predicting the maximum longitudinal and rolling shear stresses in CLT and innovative panels under out-of-plane loads are compared in this section to the FE stresses found with the numerical homogenization [1]. Dealing with the longitudinal stress, there are no stress concentration [1] and therefore the maximum value at the top or bottom lamellae can be directly identified. In contrast, the rolling shear stress shows stress concentration due to the presence of free edges [30,1]. Figure 14 shows the distribution of the $\sigma_{13}$ shear stress in the glued area of the 7-ply innovative panel from FE computations. Only half width of the

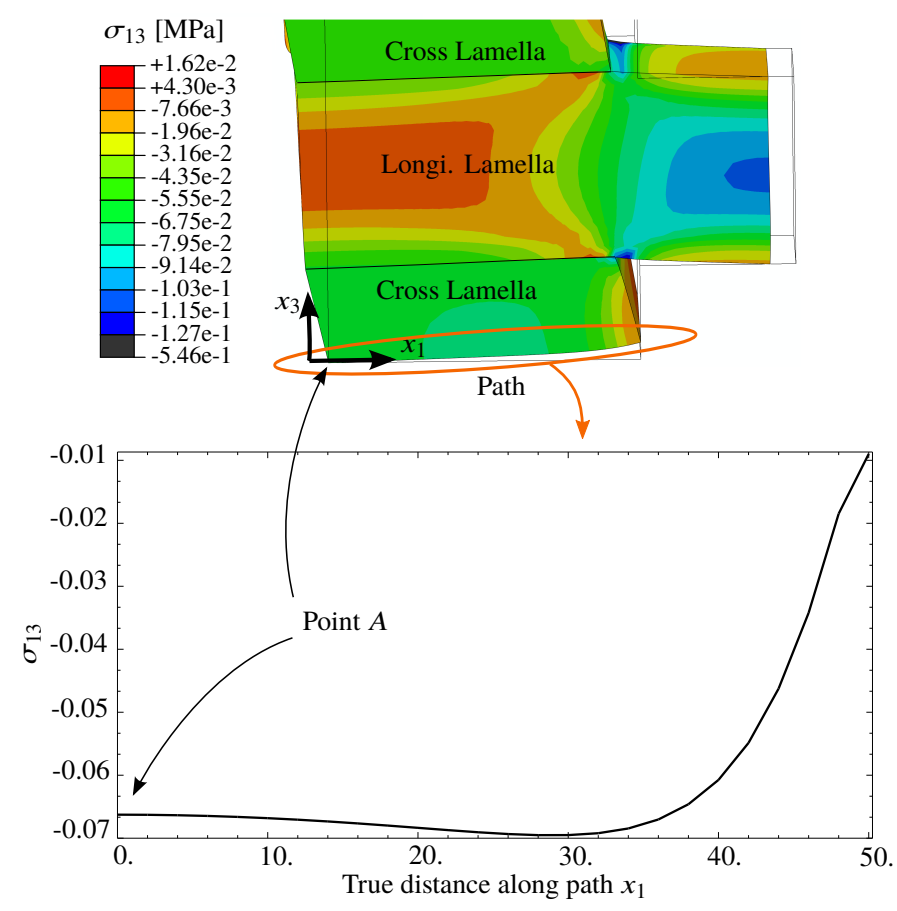

Figure 14: Distribution of rolling shear stress in the central lamella of a 7-ply $(w=100 \mathrm{~mm}, h=30 \mathrm{~mm})$

lamellae is showed. The maximum value of rolling shear stress is numerically evaluated at the central point $A$ of the lamella closest to neutral axis of the panel (Figure 14).

Figures 15 presents the relative difference between the numerical and closed-form maximum stresses as a function of gaps and for different lay-ups. The relative difference is defined as:

$$
\delta=\frac{\sigma^{\max , \text { Num }}-\sigma^{\max , \text { Closed }}}{\sigma^{\text {max }, \text { Num }}}
$$

In all figures, the relative distance does not seem fully smooth. As the closed-form formula are regular, these rather small amplitude oscillations come from the numerical discrepancy of the FE simulations. Indeed, contrary to stiffnesses which are averaged quantities in the homogenization procedure, stresses are local data more sensitive to the FE mesh.

The closed-form prediction of maximum longitudinal stress returns a very good agreement with reference numerical results, showing relative difference between $0 \%$ and $4 \%$. In particular, for a 3 -ply, the relative difference is very low, close to $1 \%$. For the 7-ply configuration, the closed-form prediction of maximum longitudinal stress can reach up to $4 \%$ of deviation from the FE reference.

For both 3-ply and 7-ply lay-ups, the closed-form prediction of maximum rolling shear stress is generally overestimated between $4 \%$ and $10 \%$. However, in the case of thick lamellae having narrow gaps the rolling shear stress is underestimated of about $4 \%$. This is because the free narrow edge generates rather non-uniform stress distributions especially when the aspect ratio is small.

Globally, the obtained relative difference between $-4 \%$ and $+10 \%$ is an acceptable approximation, considering the simplic- 

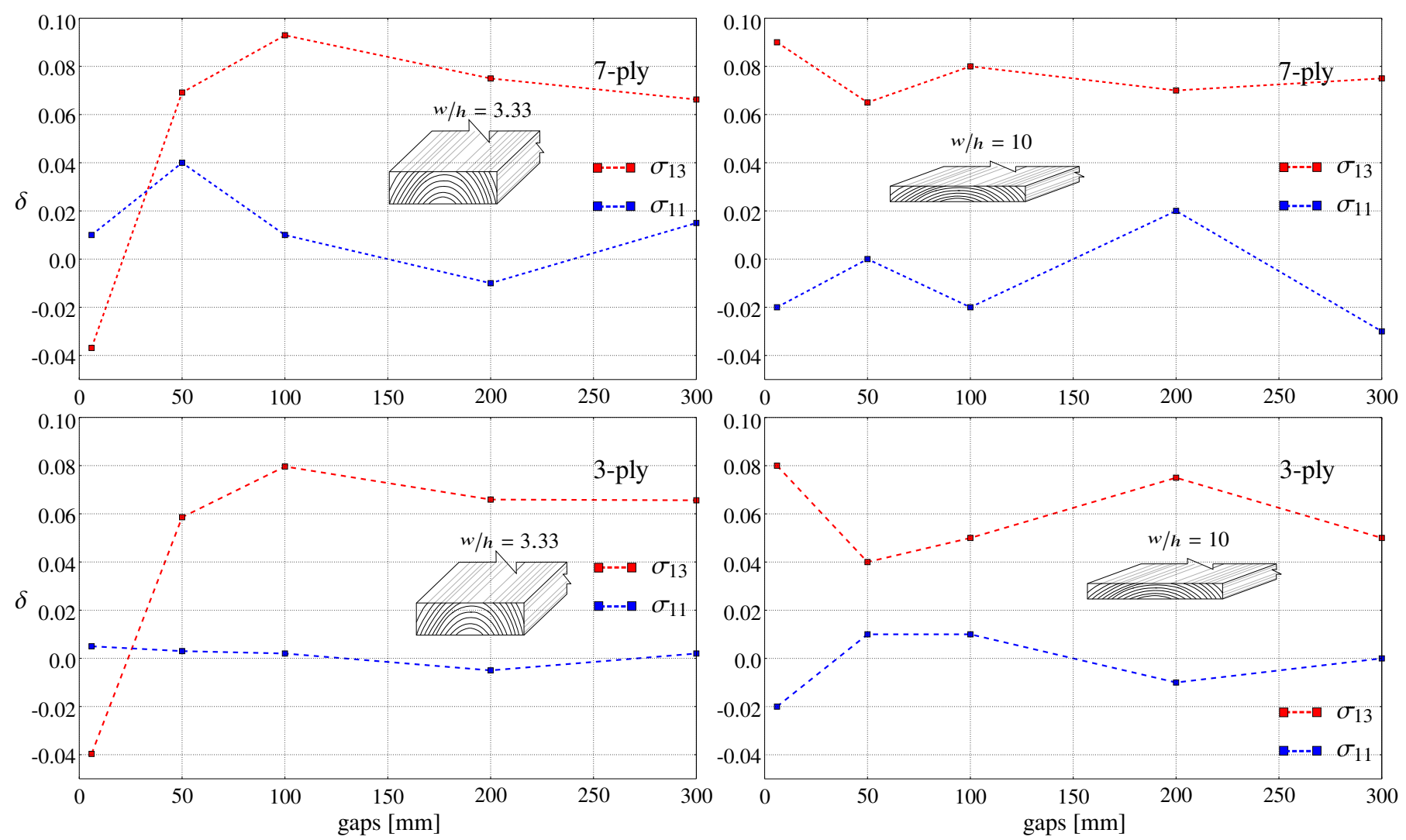

Figure 15: Relative difference $\delta$ between numerical and closed-form maximum longitudinal and rolling shear stresses in 7-ply (top) and 3-ply (bottom) panels for $w / h=3.33$ (left) and $w / h=10$ (right)

ity of use of a closed-form solution compared to FE homogenization. The prediction of longitudinal stress is accurate, while the maximum rolling shear stresses can be predicted with a slight overestimation but less than $10 \%$, that is, from an engineering point of view, on the safe side but not too conservative.

\section{Conclusion}

In this paper, closed-form solutions have been derived for predicting the elastic mechanical behavior of CLT panels and innovative timber products with wide gaps. A Reissner-Mindlin homogenization scheme has been applied to a simplified geometry of a periodic unit-cell made of beams connected by means of wooden blocks.

The final closed-form expressions for predicting bending, in-plane shear, torsional and shear force stiffnesses have been compared to the numerical homogenization. The bending stiffness expression is equivalent to the Classical Lamination Theory with volume fractions already compared in [1].

The suggested closed-form solution for the in-plane shear stiffness is in good agreement with numerical results and takes explicitly into account the number of lamellae without numerical fitting. Furthermore, it gives a prediction of in-plane shear and torsion stiffnesses similar to the method currently considered in the working draft for CLT design in Eurocode 5 [28]. When the gaps between lamellae are large, the present formulas improve these predictions.
The obtained expression of shear force compliance is based on a simplified hypothesis on the shear kinematics of the unitcell. For the 3-ply lay-up such hypothesis is satisfied and the closed-form solution for the 3-ply shear force stiffness is close to numerical results. Dealing with 5-ply and 7-ply, the closedform expression still returns an acceptable approximation of the reference shear force stiffness. Finally, the obtained closed-form expressions for estimating the maximum longitudinal and rolling shear stress acting on CLT floors with gaps show a relative distance from the reference FE results between $-4 \%$ and $+10 \%$, which is a good approximation.

The simplified expressions derived in this study can be used for predicting the elastic behavior of CLT and innovative panels with gaps in practical applications which require a thick plate model and may be used, for instance, for estimating the plate buckling strength of innovative panels [31].

\section{Appendix A. Derivation of beam stress and energy}

The beam constitutive equations for translations $\boldsymbol{u}^{i}$ and rotations $\boldsymbol{\theta}^{i}$ fields are:

$$
\left\{\begin{array}{l}
\boldsymbol{r}^{i}=\boldsymbol{F}^{i} \cdot\left(\frac{\mathrm{d} \boldsymbol{u}^{i}}{\mathrm{~d} y_{t}}+\boldsymbol{t}^{i} \times \boldsymbol{\theta}^{i}\right) \\
\boldsymbol{m}^{i}=\boldsymbol{H}^{i} \cdot \frac{\mathrm{d} \boldsymbol{\theta}^{i}}{\mathrm{~d} y_{t}}
\end{array}\right.
$$

where $\boldsymbol{r}^{i}$ and $\boldsymbol{m}^{i}$ are respectively beams' resultant forces and bending moments that describe the beam stress state. The beam 
equilibrium equations are:

$$
\left\{\begin{array}{l}
\frac{\mathrm{d} \boldsymbol{r}^{i}}{\mathrm{~d} y_{t}}+\boldsymbol{q}^{i}=0 \\
\frac{\mathrm{d} \boldsymbol{m}^{i}}{\mathrm{~d} y_{t}}+\boldsymbol{t}^{i} \times \boldsymbol{r}^{i}+\boldsymbol{l}^{i}=0
\end{array}\right.
$$

where $\boldsymbol{q}^{i}$ and $\boldsymbol{l}^{i}$ are distributed forces and torques respectively.

In order to derive the bending and membrane stiffnesses of the panel, the loads consist in imposed relative displacements and rotations taking into account periodicity conditions $\boldsymbol{u}^{\text {ext, } i}$ and $\boldsymbol{\theta}^{\text {ext, } i}$ (see Figure 5 and [14]). For longitudinal beams $i \in \mathcal{L}$, this writes as:

$$
\begin{gathered}
\boldsymbol{u}^{b, i}-\boldsymbol{u}^{0, i}=\boldsymbol{u}^{\mathrm{ext}, i}=\left(\begin{array}{c}
b\left(e_{11}+z^{i} \chi_{11}\right) \\
b\left(e_{12}+z^{i} \chi_{12}\right) \\
\frac{-b^{2}}{2} \chi_{11}
\end{array}\right)_{\left(\boldsymbol{e}_{1}, \boldsymbol{e}_{2}, \boldsymbol{e}_{3}\right)} \\
\boldsymbol{\theta}^{b, i}-\boldsymbol{\theta}^{0, i}=\boldsymbol{\theta}^{\mathrm{ext}, i}=\left(\begin{array}{c}
-b \chi_{12} \\
b \chi_{11} \\
0
\end{array}\right)_{\left(\boldsymbol{e}_{1}, \boldsymbol{e}_{2}, \boldsymbol{e}_{3}\right)}
\end{gathered}
$$

where $\boldsymbol{u}^{0, i}=\boldsymbol{u}^{i}\left(y_{t}=0\right)$ and $\boldsymbol{u}^{b, i}=\boldsymbol{u}^{i}\left(y_{t}=b\right)$ and the same for $\boldsymbol{\theta}^{i}$. For cross beams $i \in C$, this writes as:

$$
\boldsymbol{u}^{b, i}-\boldsymbol{u}^{0, i}=\boldsymbol{u}^{\mathrm{ext}, i}=\left(\begin{array}{c}
b\left(e_{12}+z^{i} \chi_{12}\right) \\
b\left(e_{22}+z^{i} \chi_{22}\right) \\
\frac{-b^{2}}{2} \chi_{22}
\end{array}\right)_{\left(\boldsymbol{e}_{1}, \boldsymbol{e}_{2}, \boldsymbol{e}_{3}\right)}
$$

and

$$
\boldsymbol{\theta}^{b, i}-\boldsymbol{\theta}^{0, i}=\boldsymbol{\theta}^{\mathrm{ext}, i}=\left(\begin{array}{c}
-b \chi_{22} \\
b \chi_{12} \\
0
\end{array}\right)_{\left(\boldsymbol{e}_{1}, \boldsymbol{e}_{2}, \boldsymbol{e}_{3}\right)}
$$

As only relative displacements are enforced between beams' extremities, the rigid motion of the unit cell needs also to be prevented. Periodicity conditions prevent the 3 rigid rotations and only 3 rigid translations needs to be restrained eventually.

Since there are no external beam loads $\boldsymbol{q}^{i}$ or $\boldsymbol{l}^{i}$, integrating Equations A.2 leads to constant resultants and linear moments:

$$
\boldsymbol{r}^{i}=\boldsymbol{r}^{0, i} \quad \boldsymbol{m}^{i}=\boldsymbol{m}^{b / 2, i}-\left(y_{t}-\frac{b}{2}\right) \boldsymbol{t}^{i} \times \boldsymbol{r}^{0, i}
$$

where $\boldsymbol{r}^{0, i}=\boldsymbol{r}^{i}\left(y_{t}=0\right)$ and $\boldsymbol{m}^{b / 2, i}=\boldsymbol{m}^{i}\left(y_{t}=b / 2\right)$ are integration constants. Substituting Equations A.7 into the constitutive equations A.1 and integrating from $y_{t}=0$ yields:

$$
\boldsymbol{\theta}^{i}-\boldsymbol{\theta}^{0, i}=\left(\boldsymbol{H}^{i}\right)^{-1} \cdot\left(y_{t} \boldsymbol{m}^{b / 2, i}+\frac{y_{t}}{2}\left(y_{t}-b\right) \boldsymbol{t}^{i} \times \boldsymbol{r}^{0, i}\right)
$$

Rewriting the above expression for $y_{t}=b$ yields the left term equal to $\boldsymbol{\theta}^{i}(b)-\boldsymbol{\theta}^{i}(0)$. This difference is equal to the imposed rotation field $\boldsymbol{\theta}^{\text {ext, } i}$ (Equations A.3 and A.5). Therefore $\boldsymbol{m}^{b / 2, i}$ writes as:

$$
\boldsymbol{m}^{b / 2, i}=\frac{\boldsymbol{H}^{i} \cdot \boldsymbol{\theta}^{\mathrm{ext}, i}}{b}
$$

The same procedure can be applied, substituting the expression of $\boldsymbol{\theta}^{i}$ found in Equation A.8 into the constitutive equation of displacements. This leads to the following expression for $\boldsymbol{r}^{0, i}$ :

$$
\boldsymbol{r}^{0, i}=\boldsymbol{P}^{i} \cdot\left[\boldsymbol{u}^{\mathrm{ext}, i}+\boldsymbol{t}^{i} \times\left(b \boldsymbol{\theta}^{0, i}+\frac{b}{2} \boldsymbol{\theta}^{\mathrm{ext}, i}\right)\right]
$$

where the operator $\boldsymbol{P}^{i}$ is, in the beam's reference frame:

$$
\boldsymbol{P}^{i}=\left(\begin{array}{ccc}
\left(E_{L} S\right)^{-1} & 0 & 0 \\
0 & \alpha_{n} & 0 \\
0 & 0 & \alpha_{3}
\end{array}\right)_{\left(\boldsymbol{t}^{i}, \boldsymbol{n}^{i}, \boldsymbol{e}_{3}\right)}
$$

with:

$$
\alpha_{n}=\frac{b}{G_{L Z} S_{n}}+\frac{b^{3}}{12 E_{L} I_{3}}
$$

and

$$
\alpha_{3}=\frac{b}{G_{L Z} S_{3}}+\frac{b^{3}}{12 E_{L} I_{n}} .
$$

Furthermore, from symmetry considerations regarding specifically the membrane and curvature loadings, it is possible to prove that:

$$
u_{1}^{0, i}=u_{2}^{0, i}=u_{3}^{0, i}=\theta_{1}^{0, i}=\theta_{2}^{0, i}=0 .
$$

Hence the only remaining unknowns are:

$$
\boldsymbol{\theta}^{0, i}=\theta_{3}^{0, i} \boldsymbol{e}_{3}
$$

Now that the resultant forces and moments of beams are determined, it is possible to write the elastic energy of each beam $i$ (Equation 1) as function of the applied relative displacements $\boldsymbol{u}^{\text {ext, } i}$ and rotations $\boldsymbol{\theta}^{\text {ext, } i}$ as well as the unknown rotations $\theta_{3}^{0, i}$ :

$$
\begin{aligned}
& U^{\text {beam }, i}\left(\theta_{3}^{0, i}\right)=\frac{1}{2}\left\{\left[\boldsymbol{u}^{\mathrm{ext}, i}+\boldsymbol{t}^{i} \times\left(\boldsymbol{\theta}^{0, i} b+\frac{b}{2} \boldsymbol{\theta}^{\mathrm{ext}, i}\right)\right] \cdot \boldsymbol{P}^{i}\right. \\
&\left.\cdot\left[\boldsymbol{u}^{\mathrm{ext}, i}+\boldsymbol{t}^{i} \times\left(\boldsymbol{\theta}^{0, i} b+\frac{b}{2} \boldsymbol{\theta}^{\mathrm{ext}, i}\right)\right]+\left[\frac{1}{b}^{t} \boldsymbol{\theta}^{\mathrm{ext}, i} \cdot \boldsymbol{H}^{i} \cdot \boldsymbol{\theta}^{\mathrm{ext}, i}\right]\right\} .
\end{aligned}
$$

\section{Appendix B. Correction of the beam length for in-plane shear and torsional stiffness}

When $K_{\theta}=\infty$ and $b=w$, Equation (18) has to return the inplane shear stiffness of a massive CLT panel without lateral gaps $A_{33}=2 N h G_{L Z}$. This is possible introducing a correction $x$ over the beam length. Furthermore, since the term related to in-plane bending $\frac{(b-w)^{3}}{12 E I_{3}}$ has to vanish when the lateral lamellae are glued $(b=w)$, the beam length under in-plane bending is set at $b-w$. The modified beam compliance $\alpha_{n}^{*}$ is $\alpha_{n}^{*}=\frac{b-x}{G_{L Z} S_{2}}+\frac{(b-w)^{3}}{12 E_{L} I_{2}}$ and the relation to satisfy can be written as:

$$
\left(\frac{2 N \frac{w-x}{G_{L Z} S_{2}}}{N^{2}-1}\right)^{-1}=2 G_{L Z} N h
$$

where the left term is Equation (18) substituting $K_{\theta}=\infty$ and $b=w$. Considering a large number of layers $(N=\infty)$ in order to overcome the influence of upper and lower free edges, one can find that the correction on the beam length under in plane shear is $x=19 w / 24$. The same correction factor can be found applying the same procedure to Equation (19) for the torsion stiffness. Finally, the corrected value $\alpha_{n}^{*}=\frac{b-19 w / 24}{G_{L Z} 5 w h / 6}+\frac{(b-w)^{3}}{E_{L} w^{3} h}$ may be used in Equations (18) and (19). 


\section{References}

[1] L. Franzoni, A. Lebée, F. Lyon, G. Forêt, Elastic behavior of Cross Laminated Timber and timber panels with regular gaps: Thick-plate modeling and experimental validation, Engineering Structures 141 (2017) 402-416.

[2] R. Brandner, G. Flatscher, A. Ringhofer, G. Schickhofer, A. Thiel, Cross Laminated Timber (CLT): overview and development, European Journal of Wood and Wood Products 74 (2016) 331-351.

[3] EN-16351, Timber Structures: Cross Laminated Timber, requirements, European Committee for Standardization, CEN, Bruxelles, Belgium, 2016.

[4] G. Hochreiner, J. Füssl, J. Eberhardsteiner, Cross Laminated Timber plates subjected to concentrated loading. Experimental identification of failure mechanisms, Strain 50 (2013) 68-71.

[5] E. I. A. Flores, K. Saavedra, J. Hinojosa, Y. Chandra, R. Das, Multi-scale modelling of rolling shear failure in cross-laminated timber structures by homogenisation and cohesive zone models, International Journal of Solids and Structures 81 (2016) 219-232.

[6] L. Franzoni, A. Lebée, F. Lyon, G. Forêt, Influence of orientation and number of layers on the elastic response and failure modes on CLT floors: modeling and parameter studies, European Journal of Wood and Wood Products (2016).

[7] R. Brandner, P. Dietsch, J. Droscher, M. Shulte-Wrede, H. Kreuzinger, M. Sieder, G. Schickhofer, S. Winter, Shear properties of Cross Laminated Timber (CLT) under in-plane load: test configuration and experimental study, in: Proceedings of the 2nd International Network on Timber Engineering Research meeting.

[8] T. Moosbrugger, W. Guggenberger, T. Bogensperger, Cross Laminated Timber wall segments under homogeneous shear with and without openings, in: Proceedings of the 9th World Conference on Timber Engineering.

[9] A. Lebée, K. Sab, A Bending-Gradient model for thick plates. Part I: Theory, International Journal of Solids and Structures 48 (2011) 28782888.

[10] A. Lebée, K. Sab, A Bending-Gradient model for thick plates, Part II: Closed-form solutions for cylindrical bending of laminates, International Journal of Solids and Structures 48 (2011) 2889-2901.

[11] A. Lebée, K. Sab, Justification of the Bending-Gradient Theory Through Asymptotic Expansions, in: H. Altenbach, S. Forest, A. Krivtsov (Eds.), Generalized Continua as Models for Materials, Springer-Verlag Berlin Heidelberg, 2013, pp. 217-236.

[12] K. Sab, A. Lebée, Homogenization of Thick and Heterogeneous Plates, Wiley-ISTE, 2015.

[13] L. Franzoni, A. Lebée, F. Lyon, G. Forêt, Bending behavior of regularly spaced CLT panels, in: WCTE 2016 - World Conference on Timber Engineering.

[14] A. Lebée, K. Sab, Homogenization of a space frame as a thick plate: Application of the Bending-Gradient theory to a beam lattice, Computers \& Structures 127 (2013) 88-101.

[15] L. Franzoni, A. Lebée, G. Forêt, F. Lyon, Advanced modeling for design helping of heterogeneous CLT panels in bending, in: International Network on Timber Engineering Research - INTER - Meeting 48, Sibenik, Croatia, pp. 1-11.

[16] O. Perret, A. Lebée, C. Douthe, K. Sab, Experimental determination of the equivalent-layer shear stiffness of CLT through four point bending of sandwich beams, submitted (2017).

[17] O. Perret, A. Lebée, C. Douthe, K. Sab, Equivalent layer stiffness of CLT: Closed-form bounds and numerical validation, submitted (2018).

[18] T. Bogensperger, T. Moosbrugger, G. Silly, Verification of CLT-plates under loads in plane, 11th World Conference on Timber Engineering 2010, WCTE 20101 (2010) 231-240.

[19] R. A. Joebstl, T. Bogensperger, G. Schickhofer, G. Jeitler, Mechanical Behaviour of Two Orthogonally Glued Boards Technical experiment to determine torsional parameters, Proceedings of the 8th World Conference on Timber Engineering (2004).

[20] H. J. Blass, R. Goerlacher, Zum Trag- und Verformungsverhalten von BrettsperrholzElementen bei Beanspruchung in Plattenebene, Bauen mit Holz 11 (2002) 30-34.

[21] G. Jeitler, Versuchstechnische ermittlung derverdrehungskenngroessen von orthogonal verklebten brettlamellen (German), TU Graz - Graz Univeristy of Technolgy, Graz, Austria, 2004.

[22] H. Kreuzinger, Plate and shell structures. A model for common calculation tools (in German), Bauen mit Holz 1 (1999) 34-39.
[23] D. I. Jouravskii, Remarques sur la résistance d'un corps prismatique et d'une pièce composée en bois ou en tôle de fer à une force perpendiculaire à leur longueur, Annales des Ponts et Chaussées 12 (1856) 328-351.

[24] L. Franzoni, Mechanical behavior of regularly spaced Cross Laminated Timber panels. Modeling and experimental validation in ambient and fire conditions, Ph.D. thesis, Université Paris-Est, 2016.

[25] C. Czaderski, R. Steiger, M. Howald, S. Olia, A. Gulzow, P. Niemz, Tests and calculations on 3-layered cross-laminated solid wood panels supported at all edges, European Journal of Wood and Wood Products 65 (2007) 383-402.

[26] P. Mestek, Cross Laminated Timber panels under concentrated loads: design with local shear reinforcement (in German), Ph.D. thesis, Munich University of Technology, 2011.

[27] G. Hochreiner, J. Füssl, E. Serrano, J. Eberhardsteiner, Influence of wooden board strength class on the performance of Cross Laminated Timber plates investigated by means of full-field deformations measurements, Strain 50 (2014) 161-173.

[28] Eurocode 5-1-1, Working draft of design of CLT in a revised Eurocode 5-1-1. Version 2015-10-30 (confidential), European Committee for Standardization, CEN, Bruxelles, Belgium, 2015.

[29] G. Silly, Numerical study on in-plane shear and torsional stiffness of Cross Laminated Timber (in German), Diplomarbeit, Graz University of Technology, 2010.

[30] T. Ehrhart, R. Brandner, G. Schickhofer, A. Frangi, Rolling Shear Properties of some European Timber Species with Focus on Cross Laminated Timber (CLT): Test Configuration and Parameter Study, in: Proceedings of the 2nd International Network on Timber Engineering Research meeting, pp. 1-15.

[31] O. Perret, A. Lebée, C. Douthe, K. Sab, The Bending-Gradient theory for the linear buckling of thick plates: Application to Cross Laminated Timber panels, International Journal of Solids and Structures 87 (2016) $139-152$. 